\title{
ON THE LACK OF DENSITY OF LIPSCHITZ MAPPINGS IN SOBOLEV SPACES WITH HEISENBERG TARGET
}

\author{
NOEL DEJARNETTE, PIOTR HAJŁASZ, ANTON LUKYANENKO, \\ AND JEREMY T. TYSON
}

\begin{abstract}
We study the question: When are Lipschitz mappings dense in the Sobolev space $W^{1, p}\left(M, \mathbb{H}^{n}\right)$ ? Here $M$ denotes a compact Riemannian manifold with or without boundary, while $\mathbb{H}^{n}$ denotes the $n$th Heisenberg group equipped with a sub-Riemannian metric. We show that Lipschitz maps are dense in $W^{1, p}\left(M, \mathbb{H}^{n}\right)$ for all $1 \leq p<\infty$ if $\operatorname{dim} M \leq n$, but that Lipschitz maps are not dense in $W^{1, p}\left(M, \mathbb{H}^{n}\right)$ if $\operatorname{dim} M \geq n+1$ and $n \leq p<n+1$. The proofs rely on the construction of smooth horizontal embeddings of the sphere $\mathbb{S}^{n}$ into $\mathbb{H}^{n}$. We provide two such constructions, one arising from complex hyperbolic geometry and the other arising from symplectic geometry. The nondensity assertion can be interpreted as nontriviality of the $n$th Lipschitz homotopy group of $\mathbb{H}^{n}$. We initiate a study of Lipschitz homotopy groups for sub-Riemannian spaces.
\end{abstract}

\section{INTRODUCTION}

In this paper we study Sobolev mappings from a compact Riemannian manifold or from a domain in Euclidean space into the Heisenberg group. The paper is motivated by recent developments in the theory of Sobolev mappings into metric spaces and, in particular, by the work of Capogna and Lin [11] on harmonic mappings into the Heisenberg group.

The main question which we investigate in this paper is the problem of density of Lipschitz mappings. In a more classical setting the question whether smooth mappings are dense in the space of Sobolev mappings between manifolds was raised by Eells and Lemaire [16. In the case of manifolds, smooth mappings are dense if and only if Lipschitz mappings are dense. For Heisenberg targets it is more natural to ask about density of Lipschitz mappings.

Received by the editors January 29, 2014 and, in revised form, April 3, 2014.

2010 Mathematics Subject Classification. Primary 46E35, 30L99; Secondary 46E40, 26B30, 53C17, 55Q40, 55Q70.

The first author acknowledges support from NSF grants DMS 0838434 "EMSW21-MCTP: Research Experiences for Graduate Students", DMS 0901620 and DMS 0900871. The first author also acknowledges the Department of Mathematics at the University of Pittsburgh for its hospitality during the academic year 2009-2010.

The second author acknowledges support from NSF grant DMS 0900871 "Geometry and topology of weakly differentiable mappings into Euclidean spaces, manifolds and metric spaces".

The third author acknowledges support from NSF grant DMS 0838434 "EMSW21-MCTP: Research Experiences for Graduate Students".

The fourth author acknowledges support from NSF grants DMS 0555869 "Nonsmooth methods in geometric function theory and geometric measure theory on the Heisenberg group" and DMS 0901620 "Geometric analysis in Carnot groups". 
Let $M$ and $N$ be compact Riemannian manifolds, $\partial N=\emptyset$. We can always assume that $N$ is isometrically embedded into a Euclidean space $\mathbb{R}^{\nu}$ (by the Nash theorem), and in this case we define the class of Sobolev mappings $W^{1, p}(M, N)$, $1 \leq p<\infty$, as follows:

$$
W^{1, p}(M, N)=\left\{u \in W^{1, p}\left(M, \mathbb{R}^{\nu}\right): u(x) \in N \text { a.e. }\right\} .
$$

The space $W^{1, p}(M, N)$ is equipped with a metric inherited from the norm in $W^{1, p}\left(M, \mathbb{R}^{\nu}\right)$. Although every Sobolev mapping $u \in W^{1, p}(M, N)$ can be approximated by smooth mappings with values into $\mathbb{R}^{\nu}$, it is not always possible to find an approximation by mappings from the space $C^{\infty}(M, N)$. A famous example of Schoen and Uhlenbeck [48], 49] illustrates the issue. Consider the radial projection map $u_{0}: \mathbb{B}^{n+1} \rightarrow \mathbb{S}^{n}$ from the closed unit ball in $\mathbb{R}^{n+1}$ onto its boundary, given by

$$
u_{0}(x)=\frac{x}{|x|} .
$$

The mapping $u_{0}$ has a singularity at the origin, but one can easily prove that $u_{0} \in W^{1, p}\left(\mathbb{B}^{n+1}, \mathbb{S}^{n}\right)$ for all $1 \leq p<n+1$. Schoen and Uhlenbeck proved that $u_{0}$ cannot be approximated by $C^{\infty}\left(\mathbb{B}^{n+1}, \mathbb{S}^{n}\right)$ mappings when $n \leq p<n+1$.

By using this construction one can prove that if $M$ is a compact Riemannian manifold of dimension at least $n+1$, then $C^{\infty}\left(M, \mathbb{S}^{n}\right)$ mappings are not dense in $W^{1, p}\left(M, \mathbb{S}^{n}\right)$ when $n \leq p<n+1$; see [8], 27]. On the other hand, Bethuel and Zheng 8 proved that for any manifold $M, C^{\infty}\left(M, \mathbb{S}^{n}\right)$ mappings are dense in $W^{1, p}\left(M, \mathbb{S}^{n}\right)$ whenever $1 \leq p<n$. Let us also mention that Schoen and Uhlenbeck [48], [49], proved that if $\infty>p \geq n=\operatorname{dim} M$, then $C^{\infty}(M, N)$ mappings are dense in $W^{1, p}(M, N)$ for any target $N$.

Since in the case of manifold targets, density of smooth maps is equivalent to density of Lipschitz maps, the above results imply the following well-known proposition

Proposition 1.1. (a) If $M$ is a compact Riemannian manifold of dimension $\operatorname{dim} M \geq n+1$, then Lipschitz mappings $\operatorname{Lip}\left(M, \mathbb{S}^{n}\right)$ are not dense in $W^{1, p}\left(M, \mathbb{S}^{n}\right)$ when $n \leq p<n+1$.

(b) If $M$ is a compact Riemannian manifold of dimension $\operatorname{dim} M \leq n$, then Lipschitz mappings $\operatorname{Lip}\left(M, \mathbb{S}^{n}\right)$ are dense in $W^{1, p}\left(M, \mathbb{S}^{n}\right)$ for all $1 \leq p<\infty$.

In general, the answer to the question of whether for the given two manifolds $M, N$ and $1 \leq p<n=\operatorname{dim} M, C^{\infty}(M, N)$ mappings are dense in $W^{1, p}(M, N)$ depends on the topological structure of the manifolds. A complete solution to the problem has been obtained by Hang and Lin 31] who corrected earlier results of Bethuel [7] and the generalized results of Hajłasz [22]. We refer the reader to the survey 27] for a detailed discussion and references.

There are several equivalent ways to define the space of Sobolev mappings with target the Heisenberg group $\mathbb{H}^{n}$. We refer to [21, 42] or [12 for the basic theory of the Heisenberg group and sub-Riemannian geometry; see also Section 2. In this paper we define such mappings using an isometric embedding of $\mathbb{H}^{n}$ into a Banach space. We equip $\mathbb{H}^{n}$ with its standard Carnot-Carathéodory metric. As we will see later on, the answer to the question of density of Lipschitz maps in spaces of Sobolev maps with metric space targets can potentially change under a bi-Lipschitz deformation of the target space. Our results hold for a variety of metrics on $\mathbb{H}^{n}$ which are bi-Lipschitz equivalent with the Carnot-Carathéodory metric. 
Every separable metric space, in particular, the Heisenberg group, can be isometrically embedded into $\ell^{\infty}$ (e.g., via the Kuratowski embedding (5.1)). Thus we may assume that $\mathbb{H}^{n} \subset \ell^{\infty}$ and for a compact Riemannian manifold $M$ (with or without boundary) we define

$$
W^{1, p}\left(M, \mathbb{H}^{n}\right)=\left\{u \in W^{1, p}\left(M, \ell^{\infty}\right): u(x) \in \mathbb{H}^{n} \text { a.e. }\right\} .
$$

The vector valued Sobolev space $W^{1, p}\left(M, \ell^{\infty}\right)$ is a Banach space and $W^{1, p}\left(M, \mathbb{H}^{n}\right)$ is equipped with the metric inherited from the norm. Our definition is different than that provided by Capogna and Lin [11, but it is equivalent; see Subsection 6.1 for details.

One may wonder if it would be possible to define the space $W^{1, p}\left(M, \mathbb{H}^{n}\right)$ via an isometric embedding of $\mathbb{H}^{n}$ into a Banach space different than $\ell^{\infty}$. Recently Cheeger and Kleiner [13, Theorem 1.6], [14, Theorem 4.2] proved that $\mathbb{H}^{n}$ does not admit a bi-Lipschitz embedding into any Banach space with the Radon-Nikodym property. Separable dual spaces and reflexive spaces (separable or not) have the Radon-Nikodym property. In view of this result there is little hope to replace $\ell^{\infty}$ by a better space in the definition of $W^{1, p}\left(M, \mathbb{H}^{n}\right)$.

As in the case of mappings between manifolds, Lipschitz mappings $\operatorname{Lip}\left(M, \ell^{\infty}\right)$ are dense in $W^{1, p}\left(M, \mathbb{H}^{n}\right)$, but the question is whether we can take the approximating sequence from $\operatorname{Lip}\left(M, \mathbb{H}^{n}\right)$. It turns out that this is not always possible.

Theorem 1.2. Equip $\mathbb{H}^{n}$ with the Carnot-Carathéodory metric.

(a) If $M$ is a compact Riemannian manifold of dimension $\operatorname{dim} M \geq n+1$, then Lipschitz mappings $\operatorname{Lip}\left(M, \mathbb{H}^{n}\right)$ are not dense in $W^{1, p}\left(M, \mathbb{H}^{n}\right)$ when $n \leq p<n+1$.

(b) If $M$ is a compact Riemannian manifold of dimension $\operatorname{dim} M \leq n$, then Lipschitz mappings $\operatorname{Lip}\left(M, \mathbb{H}^{n}\right)$ are dense in $W^{1, p}\left(M, \mathbb{H}^{n}\right)$ for all $1 \leq p<$ $\infty$.

The proof of the failure of density in the case $n \leq p<n+1$ and $\operatorname{dim} M \geq n+1$ is based on the following special case of Theorem $1.2(\mathrm{a})$.

Proposition 1.3. Lipschitz maps $\operatorname{Lip}\left(\mathbb{B}^{n+1}, \mathbb{H}^{n}\right)$ are not dense in $W^{1, p}\left(\mathbb{B}^{n+1}, \mathbb{H}^{n}\right)$ when $n \leq p<n+1$.

Once this result is proved, the case of general $M$ follows from an elementary surgery argument which allows us to build mappings from $M$ to $\mathbb{H}^{n}$ that contain the mappings constructed in Proposition 1.3 on $(n+1)$-dimensional slices.

Question 1.4. Are Lipschitz maps dense in $W^{1, p}\left(\mathbb{B}^{n+1}, \mathbb{H}^{n}\right)$ when $p \geq n+1$ or $1 \leq p<n$ ?

We have stated Theorem 1.2 for the Carnot-Carathéodory metric $d_{c c}$ on $\mathbb{H}^{n}$ because it is a natural metric associated to the sub-Riemannian structure on $\mathbb{H}^{n}$, and also because - in some sense - it is the most difficult metric on $\mathbb{H}^{n}$ to consider for this question. The conclusion of Theorem 1.2 holds also for other metrics on $\mathbb{H}^{n}$ which are bi-Lipschitz equivalent to $d_{c c}$, such as the Korányi metric (2.4). Note that results of Hajłasz 24, 26] imply that the answer to the Lipschitz density question can depend on the choice of the metric in the target, even within a bi-Lipschitz equivalence class.

Theorem 1.2 and Proposition 1.1 show an analogy between Sobolev mappings into the Heisenberg groups and mappings into spheres. The analogy would be even 
more complete, if we could answer Question 1.4 in the affirmative. In some sense $\mathbb{H}^{n}$ behaves like $\mathbb{S}^{n}$, but the analogy is quite intricate. Indeed, smooth mappings are always dense in the space of Sobolev mappings into a manifold which is diffeomorphic to $\mathbb{R}^{2 n+1}$. However, $\mathbb{H}^{n}$ is only homeomorphic to $\mathbb{R}^{2 n+1}$. Although the identity mapping from $\mathbb{H}^{n}$ to $\mathbb{R}^{2 n+1}$ is locally Lipschitz, the Heisenberg group is not even locally bi-Lipschitz homeomorphic to $\mathbb{R}^{2 n+1}$, as the Hausdorff dimension of $\mathbb{H}^{n}$ equals $2 n+2$. Thus one needs to look directly at the geometry of the Heisenberg group which is very complicated.

In the case of Sobolev mappings into manifolds, the answer to the density question depends, in particular, on homotopy groups of the target. The homotopy groups of $\mathbb{H}^{n}$ are trivial, but a more appropriate object to consider would be Lipschitz homotopy groups. For example, the $n$th Lipschitz homotopy group of $\mathbb{H}^{n}$ is nontrivial [5], 50, and this fact is principally responsible for the lack of density. We discuss Lipschitz homotopy groups and sub-Riemannian manifolds in Section 4.

Remark 1.5 (Added in December 2012). Wenger and Young [51] further develop the theory of Lipschitz homotopy groups of Heisenberg groups in relation to Lipschitz extension problems. The related paper [29] contains an alternate proof of the nontriviality of the $n$th Lipschitz homotopy group of $\mathbb{H}^{n}$ and other results of a similar nature, together with applications to Lipschitz nondensity in Sobolev spaces. See also Remark 4.18

In the proof of Proposition 1.3 a Sobolev mapping which cannot be approximated by Lipschitz mappings will be constructed as the composition of the cavitation map (1.1) with an explicit bi-Lipschitz embedding of the sphere $\mathbb{S}^{n}$ into $\mathbb{H}^{n}$. The existence of the latter is of independent interest.

Theorem 1.6. For any $n \geq 1$, there exists a bi-Lipschitz embedding of $\mathbb{S}^{n}$ into $\mathbb{H}^{n}$.

As observed by Balogh and Fässler [5] and Wenger and Young [50], the embedding from Theorem 1.6 admits no Lipschitz extension from $\mathbb{B}^{n+1}$ to $\mathbb{H}^{n}$. Thus

$$
\pi_{n}^{\text {Lip }}\left(\mathbb{H}^{n}\right) \neq 0 .
$$

To show that the mapping whose construction was described above cannot be approximated by Lipschitz mappings, we will use a result of Ambrosio and Kirchheim 3. and Magnani 38, 39 on the pure unrectifiability of $\mathbb{H}^{n}$. In the proof of density in Theorem 1.2(b), we will use recent results of Wenger and Young [50. Theorem [1.6 is also related to recent work on the Lipschitz extension problem with sub-Riemannian target [5], 47] and on the construction of Legendrian submanifolds of contact manifolds [17.

Another issue is understanding what it means for a sequence of Sobolev mappings $f_{k} \in W^{1, p}\left(M, \mathbb{H}^{n}\right)$ to converge to $f \in W^{1, p}\left(M, \mathbb{H}^{n}\right)$.

The Heisenberg group $\mathbb{H}^{n}$ is homeomorphic to $\mathbb{R}^{2 n+1}$ and the identity mapping from $\mathbb{H}^{n}$ to $\mathbb{R}^{2 n+1}$ is locally Lipschitz. Hence if $f \in W^{1, p}\left(M, \mathbb{H}^{n}\right)$ is bounded, then also $f \in W^{1, p}\left(M, \mathbb{R}^{2 n+1}\right)$. More generally, if $f \in W^{1, p}\left(M, \mathbb{H}^{n}\right)$ is not necessarily bounded, then still $f$ as a mapping into $\mathbb{R}^{2 n+1}$ is absolutely continuous on almost all lines. Moreover, the directional derivatives of $f$ are horizontal vectors and hence for $f \in W^{1, p}\left(\Omega, \mathbb{H}^{n}\right), \Omega \subset \mathbb{R}^{m}$ we can define

$$
|\nabla f|_{\mathbb{H}}=\left(\sum_{k=1}^{m}\left|\frac{\partial f}{\partial x_{k}}\right|_{\mathbb{H}}^{2}\right)^{1 / 2},
$$


where $|v|_{\mathbb{H}}$ stands for the length of the horizontal vector with respect to the given metric in the horizontal distribution. See Section 2 for definitions.

Theorem 1.7. Let $\Omega$ be a bounded domain in $\mathbb{R}^{m}$. Suppose that $f_{k}, f \in W^{1, p}\left(\Omega, \mathbb{H}^{n}\right)$, $k=1,2, \ldots, 1 \leq p<\infty$, and $f_{k} \rightarrow f$ in $W^{1, p}\left(\Omega, \mathbb{H}^{n}\right)$. Then

$$
\int_{\left\{f_{k}-f \notin Z\right\}}\left(\left|\nabla f_{k}\right|_{\mathbb{H}}^{p}+|\nabla f|_{\mathbb{H}}^{p}\right) \rightarrow 0
$$

as $k \rightarrow \infty$, where $Z$ denotes the center of $\mathbb{H}^{n}$.

Clearly the theorem generalizes to the case in which $\Omega$ is replaced by a compact manifold.

The condition is surprisingly strong. In particular, it shows that $f_{k}$ must differ from $f$ by an element of the center $Z$ on a large set. This phenomenon is quite unlike the case of manifold or Euclidean targets. Similar phenomena have been previously observed in 26 .

Since bounded functions in $W^{1, p}\left(M, \mathbb{H}^{n}\right)$ belong also to $W^{1, p}\left(M, \mathbb{R}^{2 n+1}\right)$ the proof of Theorem 1.7 gives

Corollary 1.8. Let $M$ be a compact Riemannian manifold. Suppose that $f_{k}, f \in$ $W^{1, p}\left(M, \mathbb{H}^{n}\right), k=1,2, \ldots$, are uniformly bounded (i.e., the ranges of all the mappings are contained in a fixed bounded subset of $\left.\mathbb{H}^{n}\right)$. If $f_{k} \rightarrow f$ in $W^{1, p}\left(M, \mathbb{H}^{n}\right)$, then $f_{k} \rightarrow f$ in $W^{1, p}\left(M, \mathbb{R}^{2 n+1}\right)$.

Theorem 1.7 shows, however, that the converse implication is not true. Indeed, if $f_{k}, f \in W^{1, p}\left(M, \mathbb{H}^{n}\right)$ and $f_{k} \rightarrow f$ in $W^{1, p}\left(M, \mathbb{R}^{2 n+1}\right)$, then it is very rarely true that $f_{k} \rightarrow f$ in $W^{1, p}\left(M, \mathbb{H}^{n}\right)$.

The paper is organized as follows. In Section 2 we recall the definition and basic properties of sub-Riemannian manifolds and the Heisenberg group. In Section 3 we provide two different proofs of Theorem 1.6. We find it important to present two different approaches as they refer to different geometric structures underlying $\mathbb{H}^{n}$. The first approach is based on the interpretation of $\mathbb{H}^{n}$ as a conformal image of the boundary of the unit ball in $\mathbb{C}^{n+1}$, punctured at one point, while the second is based on ideas from symplectic geometry. In Section 4 we discuss Lipschitz homotopy groups. We do not prove any deep results there, but we think that Lipschitz homotopy groups will eventually play an important role in geometric analysis and geometric topology and we would like to advertise the subject. In Section 5 we define the class of Sobolev mappings into metric spaces and in particular into the Heisenberg group. We follow the presentation given in [30. In Section 6] we prove Theorem 1.7 and Corollary 1.8, and show that the class of Sobolev mappings into the Heisenberg group defined in our paper agrees with that defined by Capogna and Lin [11. Section 7 contains the proofs of Proposition 1.3 and Theorem 1.2, Finally, Section 8 contains a variant of Theorem 1.2 where the target space is replaced by the sub-Riemannian Grushin plane.

\section{Sub-Riemannian GeOmetry}

2.1. The Heisenberg group. We represent the Heisenberg group as the space $\mathbb{H}^{n}=\mathbb{C}^{n} \times \mathbb{R}=\mathbb{R}^{2 n+1}$ equipped with the group law

$$
(z, t) *\left(z^{\prime}, t^{\prime}\right)=\left(z+z^{\prime}, t+t^{\prime}+2 \operatorname{Im} \sum_{j=1}^{n} z_{j} \overline{z_{j}^{\prime}}\right) .
$$


The Heisenberg group $\mathbb{H}^{n}$ is a Lie group. A basis of left invariant vector fields is given by

$$
X_{j}=\frac{\partial}{\partial x_{j}}+2 y_{j} \frac{\partial}{\partial t}, Y_{j}=\frac{\partial}{\partial y_{j}}-2 x_{j} \frac{\partial}{\partial t}, \quad j=1, \ldots, n,
$$

and $T=\frac{\partial}{\partial t}$. Here and henceforth we use the notation

$$
(z, t)=\left(z_{1}, \ldots, z_{n}, t\right)=\left(x_{1}, y_{1}, \ldots, x_{n}, y_{n}, t\right) .
$$

Note that $\left[X_{j}, Y_{j}\right]=-4 T, j=1, \ldots, n$, while all other Lie brackets of pairs of vectors taken from (2.1) are equal to zero. The Heisenberg group is equipped with the horizontal distribution $H \mathbb{H}^{n}$, which is defined at every point $p \in \mathbb{H}^{n}$ by

$$
H_{p} \mathbb{H}^{n}=\operatorname{span}\left\{X_{1}(p), \ldots, X_{n}(p), Y_{1}(p), \ldots, Y_{n}(p)\right\} .
$$

The distribution $H \mathbb{H}^{n}$ is equipped with a left invariant metric $\mathrm{g}$ such that the vectors

$$
X_{1}(p), \ldots, X_{n}(p), Y_{1}(p), \ldots, Y_{n}(p)
$$

are orthonormal at every point $p \in \mathbb{H}^{n}$. We denote by $o=(0,0)$ the identity element in $\mathbb{H}^{n}$. A family of anisotropic dilations $\left(\delta_{r}\right)_{r>0}$ on $\mathbb{H}^{n}$ is defined by

$$
\delta_{r}(z, t)=\left(r z, r^{2} t\right), \quad r>0 .
$$

An absolutely continuous curve $\gamma:[a, b] \rightarrow \mathbb{H}^{n}$ is called horizontal if $\gamma^{\prime}(s) \in$ $H_{\gamma(s)} \mathbb{H}^{n}$ for almost every $s$. The Heisenberg group $\mathbb{H}^{n}$ is equipped with the CarnotCarathéodory metric $d_{c c}$ which is defined as the infimum of the lengths of horizontal curves connecting two given points. The length of the curve is computed with respect to the metric $\mathrm{g}$ on $H \mathbb{H}^{n}$. Let us remark in passing at this point that we will denote by $|\cdot|_{\mathbb{H}}$ the norm on the horizontal bundle induced by the metric $\mathbf{g}$, i.e.,

$$
|v|_{\mathbb{H}}=\mathbf{g}_{p}(v, v)^{1 / 2} \quad \text { if } v \in H_{p} \mathbb{H}^{n} .
$$

It is well known that any two points in $\mathbb{H}^{n}$ can be connected by a horizontal curve and hence $d_{c c}$ is a true metric. Actually, $d_{c c}$ is topologically equivalent to the Euclidean metric. Moreover, for any compact set $K$ there is a constant $C \geq 1$ such that

$$
C^{-1}|p-q| \leq d_{c c}(p, q) \leq C|p-q|^{1 / 2}
$$

for all $p, q \in K$. In what follows $\mathbb{H}^{n}$ will always be regarded as the metric space $\left(\mathbb{H}^{n}, d_{c c}\right)$. It follows from (2.3) that the identity mapping from $\mathbb{H}^{n}$ to $\mathbb{R}^{2 n+1}$ is locally Lipschitz, but its inverse is only locally Hölder continuous with exponent $1 / 2$. The Hausdorff dimension of $\mathbb{H}^{n}$ equals $2 n+2$ since

$$
\mathcal{H}_{c c}^{2 n+2}\left(B_{c c}(p, r)\right)=C r^{2 n+2}
$$

for all $p \in \mathbb{H}^{n}$ and $r>0$. Here $\mathcal{H}_{c c}^{2 n+2}$ stands for the $(2 n+2)$-dimensional Hausdorff measure with respect to $d_{c c}$ and $B_{c c}(p, r)$ denotes a ball with respect to the CarnotCarathéodory metric. In fact, these Hausdorff measures are invariant with respect to left translation and scale correctly under the anisotropic dilations $\delta_{r}$ defined above.

The variational problem which defines the Carnot-Carathéodory metric always admits a solution: $\left(\mathbb{H}^{n}, d_{c c}\right)$ is a geodesic metric space. The geodesics can be written down explicitly in parametric form. See, for example, the books by Montgomery 42, Bellaïche [6], Capogna-Danielli-Pauls-Tyson [12] or the paper of Marenich 40] 
for details. In fact, sub-Riemannian geodesics in $\mathbb{H}^{1}$ are precisely the horizontal lifts of circular arcs in $\mathbb{R}^{2}$ solving Dido's isoperimetric problem.

As a result, there is a rather complicated implicit formula for $d_{c c}$. We will not need this formula in this paper. There are other metrics on $\mathbb{H}^{n}$ which are bi-Lipschitz equivalent to $d_{c c}$, for instance, the Korányi metric $d_{K}$ defined by

$$
d_{K}(p, q)=\left\|q^{-1} * p\right\|_{K},
$$

where $\|(z, t)\|_{K}=\left(|z|^{4}+t^{2}\right)^{1 / 4}$.

On the Heisenberg group there is a natural horizontal gradient

$$
\nabla_{\mathbb{H}} u=\sum_{j=1}^{n}\left(X_{j} u\right) X_{j}+\left(Y_{j} u\right) Y_{j}
$$

whose length with respect to the metric $\mathbf{g}$ on $H \mathbb{H}^{n}$ equals

$$
\left|\nabla_{\mathbb{H}} u\right|_{\mathbb{H}}=\sqrt{\sum_{j=1}^{n}\left|X_{j} u\right|^{2}+\left|Y_{j} u\right|^{2}}
$$

Let $\Omega \subset \mathbb{H}^{n}$ be a domain. A function $u: \Omega \rightarrow \mathbb{R}$ is said to be continuously horizontally differentiable at a point $p \in \Omega$ if $X_{j} u$ and $Y_{j} u$ are continuous at $p$ for all $1 \leq j \leq n$. We denote the class of functions on $\Omega$ which are $k$ times continuously horizontally differentiable at each point of $\Omega$ by $C_{\mathbb{H}}^{k}(\Omega)$.

The center of the Heisenberg group is the vertical axis ( $t$-axis)

$$
Z=\left\{(z, t) \in \mathbb{H}^{n}: z=0\right\} .
$$

It easily follows from the group law that, for $p, q \in \mathbb{H}^{n}$, we have

$$
q^{-1} * p \in Z \text { if and only if } p-q \in Z \text {. }
$$

For $q \in \mathbb{H}^{n}$, let $d_{q}: \mathbb{H}^{n} \rightarrow \mathbb{R}$ be the function

$$
d_{q}(p)=d_{c c}(p, q) .
$$

As with all distance functions on metric spaces, $d_{q}$ is 1-Lipschitz. By the PansuRademacher differentiation theorem [45], $d_{q}$ is horizontally differentiable at almost every point of $\mathbb{H}^{n}$. However, a stronger result holds.

Lemma 2.1. For each $q \in \mathbb{H}^{n}, d_{q}$ is in $C^{\infty}\left(\mathbb{H}^{n} \backslash\left\{p: q^{-1} * p \in Z\right\}\right)$.

Here the $C^{\infty}$ regularity refers to the underlying Euclidean structure on $\mathbb{R}^{2 n+1}$, but note that this is equivalent to $C^{\infty}$ regularity in horizontal directions only. Indeed, if $g \in C_{\mathbb{H}}^{k}(\Omega)$ for some $k \geq 1$, then it is easy to see that $g \in C^{\lfloor k / 2\rfloor}(\Omega)$, hence $C^{\infty}(\Omega)=C_{H}^{\infty}(\Omega)$.

Lemma 2.1 was explicitly proved by Monti 43 in the case of $\mathbb{H}^{1}$, and by Ambrosio-Rigot [4] in the case of $\mathbb{H}^{n}$. Note that Monti and Ambrosio-Rigot only state that $d_{q}$ is Euclidean $C^{1}$, however, the proof easily extends to yield the improved regularity asserted in Lemma 2.1.

The preceding result together with the chain rule imply that if $q_{0} \in \mathbb{H}^{n}$ and $f$ : $(a, b) \rightarrow \mathbb{H}^{n}$ is a horizontal curve, differentiable at $s_{0}$, and such that $q_{0}^{-1} * f\left(s_{0}\right) \notin Z$, then the function

is differentiable at $s_{0}$ and

$$
u(s)=d_{c c}\left(f(s), q_{0}\right)
$$

$$
u^{\prime}\left(s_{0}\right)=\left\langle\nabla_{\mathbb{H}} d_{q_{0}}\left(f\left(s_{0}\right)\right), f^{\prime}\left(s_{0}\right)\right\rangle_{\mathbb{H}} .
$$


Here, $\langle\cdot, \cdot\rangle_{\mathbb{H}}$ denotes the fixed and given metric defined on the horizontal distribution of $\mathbb{H}^{n}$.

Monti also proved that the Carnot-Carathéodory distance function satisfies the eikonal equation. See Theorem 3.8 in [43]. Expressed in the above language, this reads

Lemma 2.2 (Monti). For $p, q \in \mathbb{H}^{n}$ such that $q^{-1} * p \notin Z,\left|\nabla_{\mathbb{H}} d_{q}(p)\right|_{\mathbb{H}}=1$.

This lemma is actually an easy consequence of Lemma 2.1. Indeed, since the function $d_{q}$ is 1 -Lipschitz, $\left|\nabla_{\mathbb{H}} d_{q}(p)\right| \leq 1$. On the other hand, if $\gamma(t)$ is a geodesic parametrized by arc-length connecting $q$ to $p$ and passing through $p$ at $t=t_{0}$, then $d_{q}(\gamma(t))=t$ and hence

$$
1=\left.\frac{d}{d t}\right|_{t=t_{0}} d_{q}(\gamma(t))=\left\langle\nabla_{\mathbb{H}} d_{q}(p), \gamma\left(t_{0}\right)\right\rangle_{\mathbb{H}} \leq\left|\nabla_{\mathbb{H}} d_{q}(p)\right|_{\mathbb{H}} .
$$

Remark 2.3. The above argument (due to Monti) shows that $\left|\nabla_{\mathbb{H}} d_{q}(p)\right|$ is attained as the directional derivative in a geodesic direction. We will need this fact in the proof of Lemma 6.3.

2.2. Geometric measure theory in the Heisenberg group. The notion of rectifiability is fundamental in geometric measure theory. A (countably) $k$-rectifiable set is one which can be well approximated, in a Lipschitz sense, by subsets of $\mathbb{R}^{k}$ up to a set of Hausdorff $k$-measure zero. Dual to this is the notion of unrectifiable set. A purely $k$-unrectifiable set is one which contains no subset of positive Hausdorff $k$-measure which is the Lipschitz image of a set in $\mathbb{R}^{k}$. For subsets of Euclidean space, there is a nice dichotomy between these notions. We refer to the book of Mattila [41, Chapter 15] for details.

The notions can be extended to general metric spaces.

Definition 2.4. A metric space $(X, d)$ is countably $k$-rectifiable if there exists a countable family of subsets $A_{j} \subset \mathbb{R}^{k}$ and Lipschitz maps $f_{j}: A_{j} \rightarrow X$ so that $\mathcal{H}^{k}\left(X \backslash \bigcup_{j} f_{j}\left(A_{j}\right)\right)=0$.

A metric space $(X, d)$ is purely $k$-unrectifiable for some integer $k \geq 1$, if $\mathcal{H}^{k}(f(A))=0$ for all sets $A \subset \mathbb{R}^{k}$ and all Lipschitz maps $f: A \rightarrow X$.

Here $\mathcal{H}^{k}$ denotes $k$-dimensional Hausdorff measure in $(X, d)$.

It turns out that rectifiability, defined as above, is of limited use in sub-Riemannian spaces. One indication of this fact is the following theorem of AmbrosioKirchheim and Magnani. See [3, Theorem 7.2], 38, Proposition 4.4.2] and [39, Theorem 1.1] and compare [5, Proposition 1 and Theorem 3].

Theorem 2.5 (Ambrosio-Kirchheim, $n=1$; Magnani, arbitrary $n$ ). For all $k \geq$ $n+1$, the Heisenberg group $\mathbb{H}^{n}$ is purely $k$-unrectifiable.

In other words, $\mathcal{H}_{c c}^{k}(g(F))=0$ whenever $g$ is a Lipschitz map from an subset $F \subset \mathbb{R}^{k}$ into $\mathbb{H}^{n}$ and $k \geq n+1$. Here $\mathcal{H}_{c c}^{k}$ stands for the $k$-dimensional Hausdorff measure in the metric space $\left(\mathbb{H}^{n}, d_{c c}\right)$.

One version of the Lipschitz extension problem asks for which pairs of metric spaces $X$ and $Y$ it holds true that every partially defined Lipschitz map from a subset of $X$ into $Y$ extends to a Lipschitz map of all of $X$ into $Y$. We say that the pair $(X, Y)$ has the Lipschitz extension property if there exists a constant $C \geq 1$ so that every $L$-Lipschitz map $f: A \rightarrow Y, A \subset X$, has a $C L$-Lipschitz extension $F: X \rightarrow Y$. 
With some additional work, it follows from the pure $(n+1)$-unrectifiability of $\mathbb{H}^{n}$ that the pair $\left(\mathbb{R}^{n+1}, \mathbb{H}^{n}\right)$ does not have the Lipschitz extension property. This was proved by Balogh and Fässler [5]; see also Proposition 4.7 below. On the other hand, we note the following theorem of Gromov [21]. Gromov's proof uses the deep machinery of microflexibility; a new proof which avoids the use of this machinery (and extends the result to a more general class of Carnot groups) has recently been given by Wenger and Young [50, Theorem 1.1].

Theorem 2.6 (Gromov, Wenger-Young). Let $M$ be either a compact Riemannian $k$-manifold, with or without boundary, or $M=\mathbb{R}^{k}$, where $k \leq n$. Then the pair $\left(M, \mathbb{H}^{n}\right)$ has the Lipschitz extension property.

2.3. Sub-Riemannian manifolds. Let $M$ be a smooth and connected manifold equipped with a distribution $H M \subset T M$. We allow the possibility that $H M$ has nonconstant rank, i.e., the function $p \mapsto \operatorname{dim} H_{p} M$ is not constant. (For an example, see Section 8, )

For $i \geq 1$, let $H_{p}^{i} M$ be the subspace of $T_{p} M$ spanned by the values at $p$ of all vector fields obtained as iterated commutators of length at most $i$ of sections of $H M$. Thus

$$
\begin{gathered}
H_{p}^{2} M=(H M \oplus[H M, H M])_{p}, \\
H_{p}^{3} M=\left(H^{2} M \oplus\left[H M, H^{2} M\right]\right)_{p},
\end{gathered}
$$

and so on. For fixed $p$, we obtain a flag of subspaces

$$
(0)=: H_{p}^{0} M \subset H_{p} M \subset H_{p}^{2} M \subset H_{p}^{3} M \subset \cdots \subset T_{p} M .
$$

Definition 2.7. The pair $(M, H M)$ is said to satisfy the bracket-generating property if there exists an integer $s<\infty$ so that $H_{p}^{s} M=T_{p} M$ for all $p \in M$. In this case, we call $H M$ the horizontal distribution, $H_{p} M$ the horizontal tangent space at $p$, and we call the minimal $s$ satisfying the condition the step of the distribution.

Definition 2.8. Let $(M, H M)$ satisfy the bracket-generating property, and let $\mathbf{g}=\left(\mathbf{g}_{p}\right)$ be a smoothly varying family of inner products defined on the horizontal tangent bundle $H M$. The triple $(M, H M, \mathbf{g})$ is called a sub-Riemannian manifold. Its step is the step of the distribution.

A sub-Riemannian manifold $(M, H M, \mathbf{g})$ is regular if the function

$$
p \mapsto\left(\operatorname{dim} H_{p} M, \operatorname{dim} H_{p}^{2} M, \operatorname{dim} H_{p}^{3} M, \ldots, \operatorname{dim} H_{p}^{s} M=\operatorname{dim} M\right)
$$

is constant on $M$. For example, the Heisenberg group $\left(\mathbb{H}^{n}, H \mathbb{H}^{n}, g\right)$ is a regular sub-Riemannian manifold of step two. More generally, all Carnot groups are regular sub-Riemannian manifolds. The Grushin plane (see Section 8 ) is a nonregular subRiemannian manifold of step two.

As we did for $\mathbb{H}^{n}$, we can define a Carnot-Carathéodory metric on any subRiemannian manifold. An absolutely continuous curve $\gamma$ is called horizontal if $\gamma^{\prime}(s) \in H_{\gamma^{\prime}(s)} M$ for a.e. $s$. The length of a horizontal curve $\gamma$ is computed with respect to the metric $\mathbf{g}$ on the horizontal bundle. Define a distance function $d_{c c}$ on $M$ by infimizing the lengths of horizontal curves joining two given points. The fundamental theorem of sub-Riemannian geometry is the Chow-Rashevsky theorem. See, e.g., [42, Theorem 2.2].

Theorem 2.9 (Chow, Rashevsky). Let ( $M, H M)$ be bracket-generating. Then any two points in $M$ can be connected by a horizontal curve. Consequently, on any sub-Riemannian manifold $(M, H M, \mathbf{g}), d_{c c}$ is a metric. 
An estimate similar to (2.3) holds. Let $M=(M, H M, \mathbf{g})$ be a regular subRiemannian manifold of step $s$. Let $\tilde{\mathbf{g}}$ be any Riemannian metric on $M$. Then for each compact $K \subset M$ there exists a constant $C$ so that the estimates

$$
d_{\tilde{\mathbf{g}}}(p, q) \leq d_{c c}(p, q) \leq C d_{\tilde{\mathbf{g}}}(p, q)^{1 / s}
$$

hold for all $p, q \in K$. See, for example, Nagel-Stein-Wainger [44], Gromov [21], or Montgomery [42, Theorem 2.10].

The next notion is essential in the following section.

Definition 2.10. Let $(M, H M, \mathbf{g})$ be a sub-Riemannian manifold, and let $N$ be a smooth manifold. A horizontal embedding of $N$ into $M$ is a smooth embedding $\phi: N \rightarrow M$ such that $d \phi: T N \rightarrow H M$.

For instance, horizontal embeddings of intervals into $M$ are precisely non-selfintersecting horizontal curves.

\section{Horizontal BI-Lipschitz Embeddings}

In this section we will construct bi-Lipschitz embeddings of the sphere $\mathbb{S}^{n}$ into the Heisenberg group $\mathbb{H}^{n}$. According to the following theorem, it suffices to construct horizontal embeddings of $\mathbb{S}^{n}$. We will describe two different such embeddings.

Theorem 3.1. Let $(M, H M, \mathbf{g})$ be a sub-Riemannian manifold, let $N$ be a compact manifold, and let $\phi$ be a smooth horizontal embedding of $N$ into $M$. Let $d_{\text {ext }}$ be the restriction of the $C C$ metric on $M$ to $\phi(N)$ and let $d_{\mathrm{int}}$ be the Riemannian metric on $\phi(N)$ inherited from $\mathbf{g}$. Then $d_{\mathrm{ext}}$ is bi-Lipschitz equivalent to $d_{\mathrm{int}}$. More precisely, there exists $K \geq 1$ so that

$$
K^{-1} d_{\text {int }} \leq d_{\text {ext }} \leq d_{\text {int }}
$$

on $\phi(N)$. Furthermore, any Riemannian metric on $\phi(N)$ is bi-Lipschitz equivalent to $d_{\text {ext }}$.

Proof. Since any two Riemannian metrics on a compact manifold are bi-Lipschitz equivalent, the last part of the theorem immediately follows from the bi-Lipschitz equivalence of $d_{\text {int }}$ and $d_{\text {ext }}$.

The inequality $d_{\text {ext }} \leq d_{\text {int }}$ follows immediately from the definition of the two metrics. It remains to prove the other inequality.

Without loss of generality, we may assume that $N$ is a subset of $M$ and $\phi$ is the inclusion map. Fix $\epsilon>0$ and consider the set

$$
\left\{(p, q) \in N \times N: d_{\text {ext }}(p, q) \geq \epsilon\right\} .
$$

Define

$$
K_{1}=\sup \frac{d_{\text {int }}(p, q)}{d_{\text {ext }}(p, q)},
$$

where the supremum is taken over the set in (3.1). Observe that

$$
K_{1} \leq \frac{\operatorname{diam} N}{\epsilon}
$$

is finite.

This reduces us to a local problem. We have to show that there exists $K \geq 1$ such that

$$
K^{-1} d_{\text {int }}(p, q) \leq d_{\text {ext }}(p, q)
$$

provided $d_{\text {ext }}(p, q)<\epsilon$ and $p \neq q$. The constant $\epsilon$ will be fixed later. 
Let $\operatorname{dim} M=n$ and $\operatorname{dim} N=k$. In a neighborhood $\mathcal{U}$ of each point of $N$ there is a cubic coordinate system $\varphi: \mathcal{U} \rightarrow(-3,3)^{n}$ on $M$ with coordinate functions $x_{1}, \ldots, x_{n}$ such that

$$
\mathcal{U} \cap N=\left\{x_{k+1}=0, \ldots, x_{n}=0\right\} .
$$

Since $N$ is compact, there is a finite family of coordinate systems $\left(\mathcal{U}_{i}, \varphi_{i}\right), i=$ $1,2, \ldots, m$ as above, such that

$$
N=\bigcup_{i=1}^{m} \varphi_{i}^{-1}\left((-1,1)^{n}\right) \cap N .
$$

Let

$$
\epsilon_{i}=\inf \left\{d_{c c}(r, s): r \in \varphi_{i}^{-1}\left([-1,1]^{n}\right), s \in M \backslash \varphi_{i}^{-1}\left((-2,2)^{n}\right)\right\},
$$

and

$$
\epsilon_{0}=\min \left\{\epsilon_{1}, \ldots, \epsilon_{m}\right\}
$$

Clearly, $\epsilon_{0}>0$.

Let $p, q \in \varphi_{i}^{-1}\left((-1,1)^{n}\right) \cap N$ be such that $d_{\text {ext }}(p, q)<\epsilon_{0}$. Let $\gamma$ be a horizontal curve connecting $p$ and $q$ of length $\ell(\gamma)<2 d_{\text {ext }}(p, q)$. (Here length is computed with respect to the sub-Riemannian metric $\mathbf{g}$ on $H M$.) Then $\gamma$ is contained in $\varphi_{i}^{-1}\left((-2,2)^{n}\right)$. Indeed, any horizontal curve connecting $p$ and $q$ and not contained in $\varphi_{i}^{-1}\left((-2,2)^{n}\right)$ has length

$$
\ell(\gamma) \geq 2 \epsilon_{i} \geq 2 \epsilon_{0}>2 d_{\text {ext }}(p, q) .
$$

Let $\delta>0$ be the Lebesgue number of the covering (3.2) with respect to the metric $d_{\text {ext }}$ and let $\epsilon=\min \left\{\epsilon_{0}, \delta\right\}$. Then any two points $p, q \in N$ with $d_{\text {ext }}(p, q)<\epsilon$ satisfy $p, q \in \varphi_{i}^{-1}\left((-1,1)^{n}\right) \cap N$ for some $i=1,2, \ldots, m$, furthermore, any horizontal curve $\gamma$ connecting $p$ and $q$ of length $\ell(\gamma)<2 d_{\text {ext }}(p, q)$ is contained in $\varphi_{i}^{-1}\left((-2,2)^{n}\right)$.

A simple compactness argument shows that there is a constant $K \geq 1$ such that for any $i=1,2, \ldots, m$, any $x \in[-2,2]^{n}$ and any vector $v \in H_{\varphi_{i}^{-1}(x)} M$,

$$
K^{-1}\left\|d \varphi_{i}(v)\right\|^{2} \leq \mathbf{g}_{\varphi_{i}^{-1}(x)}(v, v) \leq K\left\|d \varphi_{i}(v)\right\|^{2} .
$$

Here $\|\cdot\|$ denotes Euclidean length of a vector in $\mathbb{R}^{n}$.

As explained above, $d_{\text {ext }}(p, q)$ is the infimum of the lengths of horizontal curves connecting $p$ and $q$ and contained in $\varphi_{i}^{-1}\left((-2,2)^{n}\right)$. Denote by $\ell_{\mathbb{R}^{n}}\left(\varphi_{i} \circ \gamma\right)$ the Euclidean length of the curve $\varphi_{i} \circ \gamma$ contained in $(-2,2)^{n}$. It follows from (3.3) that

$$
K^{-1 / 2}\left|\varphi_{i}(p)-\varphi_{i}(q)\right| \leq K^{-1 / 2} \ell_{\mathbb{R}^{n}}\left(\varphi_{i} \circ \gamma\right) \leq \ell(\gamma) \leq K^{1 / 2} \ell_{\mathbb{R}^{n}}\left(\varphi_{i} \circ \gamma\right)
$$

$$
d_{\text {ext }}(p, q) \geq K^{-1 / 2}\left|\varphi_{i}(p)-\varphi_{i}(q)\right|
$$

The line segment $\sigma$ joining $\varphi_{i}(p)$ to $\varphi_{i}(q)$ is contained in the slice $\left\{x_{k+1}=0, \ldots\right.$, $\left.x_{n}=0\right\}$ and hence $\gamma=\varphi_{i}^{-1} \circ \sigma$ is a (horizontal) curve in $N$. Now (3.4) implies

$$
d_{\text {int }}(p, q) \leq \ell(\gamma) \leq K^{1 / 2} \ell_{\mathbb{R}^{n}}(\sigma)=K^{1 / 2}\left|\varphi_{i}(p)-\varphi_{i}(q)\right|
$$

Finally (3.5) and (3.6) together yield $K^{-1} d_{\text {int }}(p, q) \leq d_{\text {ext }}(p, q)$ provided $d_{\text {ext }}(p, q)<$ $\epsilon$. The proof is complete.

Next, we state a slightly stronger version of Theorem 1.6 . 
Theorem 3.2. For any $n \geq 1$, there is a horizontal and hence bi-Lipschitz embedding $\phi: \mathbb{S}^{n} \rightarrow \mathbb{H}^{n}$.

In the next two subsections we present two different proofs of Theorem 3.2 .

3.1. Horizontal embeddings of spheres via several complex variables. The proof is split into several rather independent steps.

3.1.1. CR structure. The complex structure of $\mathbb{C}^{n+1}$ induces an operator

$$
\mathcal{J}: \mathbb{R}^{2 n+2} \rightarrow \mathbb{R}^{2 n+2}
$$

defined by

$$
\mathcal{J}\left[x_{1}, y_{1}, \ldots, x_{n+1}, y_{n+1}\right]=\left[-y_{1}, x_{1}, \ldots,-y_{n+1}, x_{n+1}\right] .
$$

If $T_{z} \mathbb{C}^{n+1}$ is the real tangent space to $\mathbb{C}^{n+1}$ at $z$, then we have the tangent operator $\mathcal{J}: T_{z} \mathbb{C}^{n+1} \rightarrow T_{z} \mathbb{C}^{n+1}$ given by

$$
\mathcal{J}\left(\sum_{j=1}^{n+1}\left(a_{j} \frac{\partial}{\partial x_{j}}+b_{j} \frac{\partial}{\partial y_{j}}\right)\right)=\sum_{j=1}^{n+1}\left(-b_{j} \frac{\partial}{\partial x_{j}}+a_{j} \frac{\partial}{\partial y_{j}}\right) .
$$

Let $\Omega \subset \mathbb{C}^{n+1}$ be a domain with smooth boundary. For $z \in \partial \Omega$ let $T_{z} \partial \Omega$ be the real tangent space. We define

$$
H_{z} \partial \Omega=T_{z} \partial \Omega \cap \mathcal{J} T_{z} \partial \Omega .
$$

This is the maximal complex subspace of $T_{z} \partial \Omega$, but we will regard it as a real space. It easily follows from a dimension argument that the real dimension of $H_{z} \partial \Omega$ is $2 n$. We will call $H \partial \Omega$ the horizontal distribution in the tangent bundle $T \partial \Omega$. It is also known as the CR structure, but we will not refer to CR structures in what follows.

3.1.2. The Siegel domain. The Siegel domain $D$ is the set of $z=\left(z_{1}, \ldots, z_{n+1}\right)$ in $\mathbb{C}^{n+1}$ such that $\operatorname{Im} z_{n+1}>\sum_{j=1}^{n}\left|z_{j}\right|^{2}$. Identifying $\left(z_{1}, \ldots, z_{n+1}\right) \in \mathbb{C}^{n+1}$ with $\left(x_{1}, y_{1}, \ldots, x_{n+1}, y_{n+1}\right) \in \mathbb{R}^{2 n+2}$, we observe that the boundary $\partial D$ is defined by the equation $r=0$, where $r(z)=\sum_{j=1}^{n}\left(x_{j}^{2}+y_{j}^{2}\right)-y_{n+1}$. Hence

$$
v=\sum_{j=1}^{n+1}\left(a_{j} \frac{\partial}{\partial x_{j}}+b_{j} \frac{\partial}{\partial y_{j}}\right)
$$

is in $T_{z} \partial D$ for some $z=\left(x_{1}, y_{1}, \ldots, x_{n+1}, y_{n+1}\right) \in \partial D$ if $d r_{z}(v)=0$, which in turn is equivalent to $b_{n+1}=2 \sum_{j=1}^{n}\left(x_{j} a_{j}+y_{j} b_{j}\right)$. Now $v \in H_{z} \partial D$ if also

$$
\mathcal{J} v=\sum_{j=1}^{n+1}\left(-b_{j} \frac{\partial}{\partial x_{j}}+a_{j} \frac{\partial}{\partial y_{j}}\right)
$$

is in $T_{z} \partial D$, i.e., $a_{n+1}=2 \sum_{j=1}^{n}\left(-x_{j} b_{j}+y_{j} a_{j}\right)$. Therefore, the horizontal distribution $H \partial D$ is generated by the vector fields

$$
\tilde{X}_{j}(z)=\frac{\partial}{\partial x_{j}}+2 y_{j} \frac{\partial}{\partial x_{n+1}}+2 x_{j} \frac{\partial}{\partial y_{n+1}}
$$

and

for $j=1,2, \ldots, n$.

$$
\widetilde{Y}_{j}(z)=\frac{\partial}{\partial y_{j}}-2 x_{j} \frac{\partial}{\partial x_{n+1}}+2 y_{j} \frac{\partial}{\partial y_{n+1}}
$$


3.1.3. The Heisenberg group. Let $\pi: \mathbb{C}^{n+1} \supset \partial D \rightarrow \mathbb{C}^{n} \times \mathbb{R}$ be the projection onto the first $2 n+1$ coordinates:

$$
\pi\left(z_{1}, \ldots, z_{n+1}\right)=\left(x_{1}, y_{1}, \ldots, x_{n+1}\right) .
$$

Then $X_{j}=d \pi_{z}\left(\widetilde{X}_{j}\right)=\partial / \partial x_{j}+2 y_{j} \partial / \partial x_{n+1}$ and $Y_{j}=d \pi_{z}\left(\widetilde{Y}_{j}\right)=\partial / \partial y_{j}-$ $2 x_{j} \partial / \partial x_{n+1}$ generate the horizontal distribution in $\mathbb{H}^{n}$. The map $\pi$ is a diffeomorphism of $\partial D$ onto $\mathbb{C}^{n} \times \mathbb{R}$ which maps $H \partial D$ onto $H \mathbb{H}^{n}$. In this way we identify the boundary of the Siegel domain with the Heisenberg group.

If $\psi: \mathbb{S}^{n} \rightarrow \partial D$ is a smooth and horizontal (with respect to $H \partial D$ ) embedding of the sphere, then $\pi \circ \psi: \mathbb{S}^{n} \rightarrow \mathbb{H}^{n}$ is a smooth and horizontal embedding into the Heisenberg group. Thus to prove Theorem 3.2 it remains to construct a smooth and horizontal embedding $\psi: \mathbb{S}^{n} \rightarrow \partial D$.

3.1.4. The unit ball. Let $B=\left\{z \in \mathbb{C}^{n+1}: \sum_{j=1}^{n+1}\left|z_{j}\right|^{2}<1\right\}$ be the unit ball in $\mathbb{C}^{n+1}$. Define the horizontal distribution $H \partial B$ as before. The boundary $\partial B$ is given by $r=0$, where $r(z)=\sum_{j=1}^{n+1}\left(x_{j}^{2}+y_{j}^{2}\right)-1$, hence

$$
v=\sum_{j=1}^{n+1}\left(a_{j} \frac{\partial}{\partial x_{j}}+b_{j} \frac{\partial}{\partial y_{j}}\right)
$$

is in $T_{z} \partial B$ if $d r_{z}(v)=0$, i.e., $\sum_{j=1}^{n+1}\left(a_{j} x_{j}+b_{j} y_{j}\right)=0$.

Let $\mathbb{R}^{n+1}$ be the real subspace of $\mathbb{C}^{n+1}$ generated by the coordinates $x_{1}, \ldots, x_{n+1}$. Then $\mathbb{S}^{n}=\partial B \cap \mathbb{R}^{n+1}$ is the standard unit sphere.

Lemma 3.3. For any $z \in \mathbb{S}^{n}, T_{z} \mathbb{S}^{n} \subset H_{z} \partial B$, i.e., the sphere $\mathbb{S}^{n}$ is horizontally embedded into $\partial B$.

Proof. As before, $v=\sum_{j=1}^{n+1} a_{j} \frac{\partial}{\partial x_{j}} \in T_{z} \mathbb{S}^{n} \subset T_{z} \partial B$ if $\sum_{j=1}^{n+1} a_{j} x_{j}=0$. It remains to show that $\mathcal{J} v \in T_{z} \partial B$. We have $\mathcal{J} v=\sum_{j=1}^{n+1} a_{j} \frac{\partial}{\partial y_{j}}$. Since $y_{j}=0$ for $j=1, \ldots, n+1$ we have $\sum_{j=1}^{n+1} a_{j} y_{j}=0$, so $\mathcal{J} v \in T_{z} \partial B$.

Note that the south pole $p_{0}=\left(z_{1}, \ldots, z_{n}, z_{n+1}\right)=(0,0, \ldots,-1)$ belongs to $\mathbb{S}^{n}$. Let $R \in U(n+1)$ be a $\mathbb{C}$-linear rotation of $\mathbb{C}^{n+1}$ so that $p_{0} \notin R\left(\mathbb{S}^{n}\right)$. Since $R$ maps $\mathbb{C}$-linear subspaces of $T_{p} \mathbb{C}^{n+1}$ onto $\mathbb{C}$-linear subspaces of $T_{R(p)} \mathbb{C}^{n+1}$, it preserves the horizontal distribution $H \partial B$. Thus $R\left(\mathbb{S}^{n}\right)$ is a horizontally embedded sphere in $\partial B$ which does not contain the south pole.

3.1.5. The Cayley transform. The unit ball $B$ and the Siegel domain $D$ are biholomorphically equivalent via the Cayley transform $C: B \rightarrow D$,

$$
C\left(z_{1}, \ldots, z_{n+1}\right)=\left(\frac{z_{1}}{1+z_{n+1}}, \ldots, \frac{z_{n}}{1+z_{n+1}}, \mathbf{i}\left(\frac{1-z_{n+1}}{1+z_{n+1}}\right)\right) .
$$

The mapping $C$ extends to the boundaries. It has one singularity on the boundary. Namely, it maps the south pole $p_{0}$ into a point at infinity. Since $d C$ is $\mathbb{C}$-linear, it maps $H \partial B$ onto $H \partial D$, except at $p_{0}$. Hence $C\left(R\left(\mathbb{S}^{n}\right)\right)$ is a horizontally embedded sphere into $\partial D$. If we use the explicit rotation $R \in U(n+1)$ given by $R\left(z_{1}, \ldots, z_{n+1}\right)=\left(z_{1}, \ldots, z_{n}, \mathbf{i} z_{n+1}\right)$ and the map $\pi$ defined in (3.7), then we obtain the following map $\phi=\pi \circ C \circ R$ from $\mathbb{S}^{n}$ to $\mathbb{H}^{n}$ :

$$
\phi\left(x_{1}, \ldots, x_{n+1}\right)=\left(\frac{x_{1}}{1+\mathbf{i} x_{n+1}}, \ldots, \frac{x_{n}}{1+\mathbf{i} x_{n+1}}, \operatorname{Im}\left(\frac{\mathbf{i} x_{n+1}-1}{1+\mathbf{i} x_{n+1}}\right)\right),
$$

for $\left(x_{1}, \ldots, x_{n+1}\right) \in \mathbb{S}^{n}$. 
This completes the first construction for Theorem 3.2 .

3.2. Horizontal embeddings of spheres via symplectic geometry. In this section we indicate another construction of a smooth horizontal embedding of $\mathbb{S}^{n}$ into $\mathbb{H}^{n}$ arising from symplectic geometry. This example has previously been considered by Eckholm, Etnyre and Sullivan [17, Example 3.1] and Balogh and Fässler [5. Section 4].

We begin by recalling some background on Lagrangian and Legendrian embeddings. Consider the Euclidean space $\mathbb{R}^{2 n}$ of dimension $2 n$. We denote points in $\mathbb{R}^{2 n}$ by $\left(x_{1}, y_{1}, \ldots, x_{n}, y_{n}\right)$. Let $\omega=\sum_{j=1}^{n} d x_{j} \wedge d y_{j}$ denote the standard symplectic form. We note that $\omega$ is exact, since $\omega=\frac{1}{2} d \beta$, where $\beta=\sum_{j}\left(x_{j} d y_{j}-y_{j} d x_{j}\right)$.

A smooth mapping $f$ from an $m$-dimensional manifold $M$ into $\mathbb{R}^{2 n}$ is called Lagrangian if $f^{*} \omega=0$. If $f=\left(g_{1}, h_{1}, \ldots, g_{n}, h_{n}\right)$, this condition reads

$$
\sum_{j=1}^{n} d g_{j} \wedge d h_{j}=0
$$

In local coordinates $\left(u_{1}, \ldots, u_{m}\right)$ on $M, 3.9$ reads

$$
\sum_{\substack{k, \ell=1 \\ k<\ell}}^{m}\left(\sum_{j=1}^{n} \frac{\partial g_{j}}{\partial u_{k}} \frac{\partial h_{j}}{\partial u_{\ell}}-\frac{\partial g_{j}}{\partial u_{\ell}} \frac{\partial h_{j}}{\partial u_{k}}\right) d u_{k} \wedge d u_{\ell}=0 .
$$

Now consider Euclidean space $\mathbb{R}^{2 n+1}$ of dimension $2 n+1$. We denote points in $\mathbb{R}^{2 n+1}$ by $\left(x_{1}, y_{1}, \ldots, x_{n}, y_{n}, t\right)$. We introduce the contact form

$$
\alpha=d t+2 \sum_{j}\left(x_{j} d y_{j}-y_{j} d x_{j}\right)=d t+2 \beta .
$$

Observe that $d \alpha=4 \omega$.

A map $F: M \rightarrow \mathbb{R}^{2 n+1}$ is called Legendrian if $F^{*} \alpha=0$. If $F=(f, \tau)$ this condition reads

$$
d \tau+2 f^{*} \beta=0 .
$$

In this case, we say that $F: M \rightarrow \mathbb{R}^{2 n+1}$ is a Legendrian lift of $f: M \rightarrow \mathbb{R}^{2 n}$.

Observe that if $F$ is Legendrian, then $f$ is necessarily Lagrangian, because

$$
f^{*} \omega=\frac{1}{2} f^{*}(d \beta)=-\frac{1}{4} d^{2} \tau=0 .
$$

The following lemma is an immediate consequence of (3.11).

Lemma 3.4. Let $f: M \rightarrow \mathbb{R}^{2 n}$ be a smooth mapping. Then there exists a Legendrian lift $F$ of $f$ if and only if the 1 -form $f^{*} \beta$ is exact.

Remark 3.5. Identify $\mathbb{R}^{2 n+1}$ with the Heisenberg group $\mathbb{H}^{n}$. The kernel of $\alpha$ at a point $p$ is the horizontal subspace $H_{p} \mathbb{H}^{n}$. A map $F: M \rightarrow \mathbb{R}^{2 n+1}$ is Legendrian if and only if it is horizontal, as a map to $\mathbb{H}^{n}$.

We now recall Example 3.1 from [17.

Example 3.6. Consider the mapping $\tilde{f}: \mathbb{R}^{n+1} \rightarrow \mathbb{R}^{2 n}$ given by

$$
\tilde{f}\left(x_{0}, x^{\prime}\right)=\tilde{f}\left(x_{0}, x_{1}, \ldots, x_{n}\right)=\left(x_{1}, x_{0} x_{1}, \ldots, x_{n}, x_{0} x_{n}\right),
$$


i.e., $\tilde{f}=\left(g_{1}, h_{1}, \ldots, g_{n}, h_{n}\right)$, where $g_{j}=x_{j}, h_{j}=x_{0} x_{j}$. Here $x^{\prime}=\left(x_{1}, \ldots, x_{n}\right) \in$ $\mathbb{R}^{n}$. The rank of the derivative $d \tilde{f}$ equals $n+1$ everywhere except on the line $x^{\prime}=0$. If we restrict $\tilde{f}$ to the unit sphere $\mathbb{S}^{n}$, then, clearly, the rank of the derivative of

$$
f=\left.\tilde{f}\right|_{\mathbb{S}^{n}}: \mathbb{S}^{n} \rightarrow \mathbb{R}^{2 n}
$$

equals $n$ at all points different than $( \pm 1,0, \ldots, 0)$. However, one can easily check that also at the points $( \pm 1,0, \ldots, 0)$ the derivative of $\tilde{f}$ restricted to the tangent space of $\mathbb{S}^{n}$ has rank $n$.

This is to say that the map $f: \mathbb{S}^{n} \rightarrow \mathbb{R}^{2 n}$ is an immersion. It is not an embedding since $f( \pm 1,0, \ldots, 0)=(0, \ldots, 0)$. However, $f$ becomes an embedding when restricted to $\mathbb{S}^{n} \backslash\{(-1,0, \ldots, 0),(1,0, \ldots, 0)\}$. Note that

$$
\tilde{f}^{*} \beta=\sum_{j=1}^{n}\left(g_{j} d h_{j}-h_{j} d g_{j}\right)=\left(\sum_{j=1}^{n} x_{j}^{2}\right) d x_{0} .
$$

The form $\tilde{f}^{*} \beta$ is not exact, not even closed. On the other hand, the form

$$
\left(1-x_{0}^{2}\right) d x_{0}=d\left(x_{0}-\frac{x_{0}^{3}}{3}\right)
$$

is exact on $\mathbb{R}^{n+1}$ and coincides with $\tilde{f}^{*} \beta$ on $\mathbb{S}^{n}$, since $1-x_{0}^{2}=\sum_{j=1}^{n} x_{j}^{2}$ on $\mathbb{S}^{n}$. If $\iota: \mathbb{S}^{n} \rightarrow \mathbb{R}^{n+1}$ is the identity map, then $f=\tilde{f} \circ \iota$ and

$$
f^{*} \beta=(\tilde{f} \circ \iota)^{*} \beta=\iota^{*}\left(\tilde{f}^{*} \beta\right)=\iota^{*}\left(d\left(x_{0}-\frac{x_{0}^{3}}{3}\right)\right)=d\left(\left(x_{0}-\frac{x_{0}^{3}}{3}\right) \circ \iota\right) .
$$

Hence the form $f^{*} \beta$ is exact and thus the mapping $f: \mathbb{S}^{n} \rightarrow \mathbb{R}^{2 n}$ has a Legendrian lift

$$
F: \mathbb{S}^{n} \rightarrow \mathbb{R}^{2 n+1} .
$$

If $F=(f, \tau)$, then (3.11) gives $\tau=\frac{2}{3} x_{0}^{3}-2 x_{0}+C$. The Legendrian lift $F$ is a smooth embedding, since $\tau(-1,0, \ldots, 0) \neq \tau(1,0, \ldots, 0)$. Therefore $F$ is a horizontal embedding of $\mathbb{S}^{n}$ into $\mathbb{H}^{n}$ and thus bi-Lipschitz.

This completes the second construction for Theorem 3.2 .

Remark 3.7. Balogh and Fässler [5] show that the map $F: \mathbb{S}^{n} \rightarrow \mathbb{H}^{n}$ given in (3.13) has no Lipschitz extension $\tilde{F}: \mathbb{B}^{n+1} \rightarrow \mathbb{H}^{n}$. In fact, they show that any continuous extension $\hat{f}: \mathbb{B}^{n+1} \rightarrow \mathbb{R}^{2 n}$ of the map $f$ from (3.12) has the property that $\hat{f}\left(\mathbb{B}^{n+1}\right)$ has positive $(n+1)$-dimensional (Euclidean) Hausdorff measure $\mathcal{H}^{n+1}$. If $\tilde{F}$ were a Lipschitz extension of $F$, and $\hat{f}$ its projection onto $\mathbb{R}^{2 n}$, then we would have $\mathcal{H}_{c c}^{n+1}\left(\tilde{F}\left(\mathbb{B}^{n+1}\right)\right) \geq \mathcal{H}^{n+1}\left(\hat{f}\left(\mathbb{B}^{n+1}\right)\right)>0$, but this contradicts the pure $(n+1)$-unrectifiability of $\mathbb{H}^{n}$ as asserted in the theorem of Ambrosio-Kirchheim and Magnani (see Theorem 2.5). We generalize this result below in Proposition 4.7.

Remark 3.8. A manifold $M$ is stably parallelizable if $M \times \mathbb{R}$ is parallelizable, i.e., has trivial tangent bundle. Examples of stably parallelizable manifolds include all orientable hypersurfaces (with or without boundary) as well as all products of spheres. According to a theorem of Gromov [20, p. 61], every stably parallelizable $n$-manifold $M$ admits a Legendrian embedding into $\mathbb{R}^{2 n+1}$. According to Remark 3.5. such an embedding is horizontal when considered as a map to $\mathbb{H}^{n}$. This observation leads to numerous other horizontal embeddings of smooth manifolds into the Heisenberg 
group $\mathbb{H}^{n}$. The case of a product of spheres, $M=\mathbb{S}^{k_{1}} \times \cdots \times \mathbb{S}^{k_{r}}$, can be done explicitly using a variant of the immersion in Example 3.6

\section{HorizontAl AND LiPsCHITZ HOMOTOPY GROUPS}

For Riemannian manifolds $M, N$, the density of $C^{\infty}(M ; N)$ in $W^{1, p}(M ; N)$ depends on the topology of the two manifolds. For example, for $1 \leq p<n+1$, $C^{\infty}\left(\mathbb{B}^{n+1}, N\right)$ is dense in $W^{1, p}\left(\mathbb{B}^{n+1}, N\right)$ if and only if $\pi_{\lfloor p\rfloor}(N)=0$. See Bethuel [7] and Hang-Lin [31.

Theorem 1.2 seems to contradict a similar statement for maps $\mathbb{B}^{n+1} \rightarrow \mathbb{H}^{n}$, since $\mathbb{H}^{n} \cong \mathbb{R}^{2 n+1}$ is contractible and has $\pi_{i}\left(\mathbb{H}^{n}\right)=0$ for $i \geq 1$. However, classical homotopy groups do not take into account the metric structure of $\mathbb{H}^{n}$. We propose two modifications of the classical homotopy groups which may be appropriate for consideration of the Lipschitz density problem in sub-Riemannian manifolds and general metric spaces.

Definition 4.1. Let $\left(X, x_{0}\right)$ be a pointed metric space. We define Lipschitz homotopy groups

$$
\pi_{n}^{\operatorname{Lip}}\left(X, x_{0}\right)
$$

in the same way as classical homotopy groups, with the exception that both the maps and homotopies are required to be Lipschitz. We emphasize that we make no restriction on the Lipschitz constants. In particular, we do not require that the optimal Lipschitz constant for a homotopy between two pointed maps $f, g$ : $\left(Q^{n}, \partial Q^{n}\right) \rightarrow\left(X, x_{0}\right)$, where $Q^{n}=[0,1]^{n}$, agree with-or even be comparable to - that of the maps $f$ and $g$.

Unlike the case for the classical homotopy groups, it is not immediately clear whether the Lipschitz homotopy groups $\pi_{n}^{\operatorname{Lip}}\left(X, x_{0}\right)$ can equivalently be defined via homotopy classes of maps $\left(\mathbb{S}^{n}, s_{0}\right) \rightarrow\left(X, x_{0}\right)$ for a basepoint $s_{0} \in \mathbb{S}^{n}$. Nevertheless, one can easily check that $\pi_{n}^{\text {Lip }}\left(X, x_{0}\right)=0$ if and only if every Lipschitz map $\left(\mathbb{S}^{n}, s_{0}\right) \rightarrow\left(X, x_{0}\right)$ admits a Lipschitz extension $\mathbb{B}^{n+1} \rightarrow X$. In fact, if $\varphi: \mathbb{S}^{n} \rightarrow X$ is a Lipschitz map with no Lipschitz extension $\tilde{\varphi}: \mathbb{B}^{n+1} \rightarrow X$ and $H: Q^{n} \rightarrow \mathbb{S}^{n}$ is Lipschitz with $\left.H\right|_{\partial Q^{n}}=s_{0}$, then $\varphi \circ H$ defines a nonzero element of $\pi_{n}^{\text {Lip }}(X)$. We will use this observation in what follows to show that various Lipschitz homotopy groups of Heisenberg groups are or are not trivial.

If $\left(Y, y_{0}\right)$ is a pointed sub-Riemannian manifold, we may instead require the maps and homotopies to be smooth and horizontal. The resulting horizontal homotopy groups are called smooth horizontal homotopy groups; we denote them by

$$
\pi_{n}^{\mathrm{H}}\left(Y, y_{0}\right) .
$$

In order to guarantee that the addition in $\pi_{n}^{\mathrm{H}}\left(Y, y_{0}\right)$ results in a smooth mapping we require that the mappings in question are constant in a neighborhood of $\partial Q^{n}$. Observe, however, that any smooth and horizontal mapping

$$
f:\left(Q^{n}, \partial Q^{n}\right) \rightarrow\left(Y, y_{0}\right)
$$

is horizontally homotopic to a mapping that is constant in a neighborhood of $\partial Q^{n}$ inside $Q^{n}$.

The standard verification that homotopy groups are in fact groups applies directly to $\pi_{n}^{\mathrm{Lip}}\left(X, x_{0}\right)$ and, with smoothing, to $\pi_{n}^{\mathrm{H}}\left(Y, y_{0}\right)$. Theorem 4.2 is derived via standard arguments (see e.g. [32]). Recall that sub-Riemannian manifolds are horizontally connected according to the Chow-Rashevsky theorem (Theorem 2.9). 
It follows from the proof of the Chow-Rashevsky theorem that horizontal curves connecting given two points are piecewise smooth, but one can actually connect any two points by smooth horizontal curves; see Gromov [21, 1.2B]. This fact is needed in part (3) of the next result.

Theorem 4.2. Let $X$ be a metric space with $x_{0}, x_{1} \in X$, and $Y$ a sub-Riemannian space with $y_{0}, y_{1} \in Y$.

(1) $\pi_{n}^{\mathrm{Lip}}\left(X, x_{0}\right)$ and $\pi_{n}^{\mathrm{H}}\left(Y, y_{0}\right)$ are abelian for $n>1$.

(2) If $x_{0}$ and $x_{1}$ are joined by a rectifiable curve, then $\pi_{n}^{\mathrm{Lip}}\left(X, x_{0}\right) \cong \pi_{n}^{\mathrm{Lip}}\left(X, x_{1}\right)$.

(3) For any $y_{0}, y_{1} \in Y, \pi_{n}^{\mathrm{H}}\left(Y, y_{0}\right) \cong \pi_{n}^{\mathrm{H}}\left(Y, y_{1}\right)$.

If $X$ is rectifiably connected, we may refer to $\pi_{n}^{\text {Lip }}(X)$ without reference to basepoint as it is well-defined up to isomorphism. Likewise, $\pi_{n}^{\mathrm{H}}(Y)$ is well-defined up to isomorphism.

Theorem 4.3. Let $Y$ be a Riemannian manifold. Then $\pi_{n}(Y)=\pi_{n}^{\mathrm{Lip}}(Y)=$ $\pi_{n}^{\mathrm{H}}(Y)$ for any $n \geq 1$.

Indeed, any continuous map $f: Q^{n} \rightarrow Y$ is homotopic to a smooth one and smooth maps $f, g: Q^{n} \rightarrow Y$ that are continuously homotopic are smoothly homotopic.

Here is another situation where the Lipschitz and classical homotopy groups agree.

Example 4.4. By a result of Cannon-Conner-Zastrow [10, any continuous map from $\mathbb{S}^{n}, n \geq 2$ into $\mathbb{R}^{2}$ is homotopic to a constant map within its image. Put in other words, every planar set $X$ is aspherical; its homotopy groups $\pi_{n}(X)$ are trivial for all $n \geq 2$. According to an unpublished result of Jason Miller [33, Remark 2.14], the same conclusion holds for Lipschitz homotopy groups: $\pi_{n}^{\operatorname{Lip}}\left(X, x_{0}\right)=0$ for all $n \geq 2$ and $x_{0} \in X$ if $X \subset \mathbb{R}^{2}$.

However, Lipschitz homotopy groups can differ substantially from their classical counterparts.

Example 4.5. Let $X$ be a metric space in which no two distinct points can be connected by a rectifiable curve. Then $\pi_{n}^{\text {Lip }}\left(X, x_{0}\right)=0$ for any $x_{0} \in X$ and $n \geq 1$. Indeed, any Lipschitz map from $\mathbb{S}^{n}$ to $X$ must be constant. For example, if $X$ is a standard closed von Koch snowflake, then $\pi_{1}^{\text {Lip }}\left(X, x_{0}\right)=0$. On the other hand $\pi_{1}\left(X, x_{0}\right)=\mathbb{Z}$, since $X$ is homeomorphic to $\mathbb{S}^{1}$. Note that there is no contradiction with the previous example, as both $\pi_{n}^{\text {Lip }}\left(X, x_{0}\right)$ and $\pi_{n}\left(X, x_{0}\right)$ vanish for this (planar) set $X$ whenever $n \geq 2$.

In the original preprint version of this paper, we posed the following question. Wenger and Young [51] answered Question 4.6] in the negative.

Question 4.6. For a sub-Riemannian manifold $Y$, the inclusion $C^{\infty}\left(\mathbb{S}^{n}, Y\right) \hookrightarrow$ $\operatorname{Lip}\left(\mathbb{S}^{n}, Y\right)$ induces a homomorphism $\pi_{n}^{\mathrm{H}}(Y) \rightarrow \pi_{n}^{\text {Lip }}(Y)$. Is it an isomorphism?

We now show that smooth horizontal embeddings of $\mathbb{S}^{n}$ into $\mathbb{H}^{n}$ are not Lipschitz null-homotopic. We conclude that $\pi_{n}^{\mathrm{Lip}}\left(\mathbb{H}^{n}\right)$ and $\pi_{n}^{\mathrm{H}}\left(\mathbb{H}^{n}\right)$ are nontrivial, even though $\pi_{k}\left(\mathbb{H}^{n}\right)=0$ for $k \geq 1$.

Proposition 4.7. Let $\phi: \mathbb{S}^{n} \hookrightarrow \mathbb{H}^{n}$ be any smooth horizontal embedding. Then $\phi$ cannot be extended to a Lipschitz map $\tilde{\phi}: \mathbb{B}^{n+1} \rightarrow \mathbb{H}^{n}$. 
Proof. Balogh and Fässler [5] established the conclusion for the specific map $\phi$ : $\mathbb{S}^{n} \rightarrow \mathbb{H}^{n}$ described in Subsection 3.2 . Since in our situation we do not have an explicit representation of $\phi$, the argument has to be more general.

Assume by way of contradiction that $\tilde{\phi}: \mathbb{B}^{n+1} \rightarrow \mathbb{H}^{n}$ is a Lipschitz extension of $\phi$. According to the theorem of Ambrosio-Kirchheim and Magnani, $\mathcal{H}_{c c}^{n+1}\left(\tilde{\phi}\left(\mathbb{B}^{n+1}\right)\right)=$ 0 . Since the identity map from $\mathbb{H}^{n}$ to $\mathbb{R}^{2 n+1}$ is locally Lipschitz we also have that the $(n+1)$-dimensional Euclidean Hausdorff measure of $\tilde{\phi}\left(\mathbb{B}^{n+1}\right)$ is zero. Therefore it suffices to prove

Proposition 4.8. If $\psi: \mathbb{S}^{n} \rightarrow \mathbb{R}^{k}, k \geq n+1$ is a smooth embedding and $\tilde{\psi}$ : $\mathbb{B}^{n+1} \rightarrow \mathbb{R}^{k}$ is a Lipschitz extension of $\psi$, then $\mathcal{H}^{n+1}\left(\tilde{\psi}\left(\mathbb{B}^{n+1}\right)\right)>0$.

Proof. We will need a version of the Stokes theorem for Lipschitz maps.

Lemma 4.9. If $g: \mathbb{B}^{n+1} \rightarrow \mathbb{R}^{\ell}, \ell \geq n$, is Lipschitz and $\omega$ is a smooth $n$-form on $\mathbb{R}^{\ell}$, then

$$
\int_{\partial \mathbb{B}^{n+1}} g^{*} \omega=\int_{\mathbb{B}^{n+1}} g^{*}(d \omega) .
$$

Proof. Extend $g$ to $\tilde{g}$ on $\mathbb{B}^{n+1}(2)$ by letting

$$
\tilde{g}(x)=g\left(\frac{x}{|x|}\right) \quad \text { when }|x| \geq 1 .
$$

Thus $\tilde{g}$ is a radial extension of $g$ from $\partial \mathbb{B}^{n+1}$ to $\mathbb{B}^{n+1}(2) \backslash \mathbb{B}^{n+1}(1)$ and $\tilde{g}=g$ on $\mathbb{B}^{n+1}(1)$. Since $\tilde{g}$ on $\mathbb{B}^{n+1}(2) \backslash \mathbb{B}^{n+1}(1)$ is constant in radial directions, the rank of $d \tilde{g}$ is at most $n$ on $\mathbb{B}^{n+1}(2) \backslash \mathbb{B}^{n+1}(1)$. Thus for any $1<r<2$,

So

$$
\int_{\mathbb{B}^{n+1}(r) \backslash \mathbb{B}^{n+1}(1)} \tilde{g}^{*}(d \omega)=0,
$$

$$
\int_{\mathbb{B}^{n+1}(r)} \tilde{g}^{*}(d \omega)=\int_{\mathbb{B}^{n+1}(1)} g^{*}(d \omega) .
$$

The mapping $\tilde{g}$ is Lipschitz continuous and hence in any Sobolev space $W^{1, p}$. Fix $n+1<p<\infty$ and let $h_{i}$ be a standard approximation of $\tilde{g}$ constructed with the help of convolution. Then $h_{i} \rightarrow \tilde{g}$ in $W^{1, p}\left(\mathbb{B}^{n+1}(2)\right)$. Since $h_{i}$ is smooth, for any $0<r<2$ we have

$$
\int_{\mathbb{B}^{n+1}(r)} h_{i}^{*}(d \omega)=\int_{\mathbb{S}^{n}(r)} h_{i}^{*} \omega
$$

by the classical Stokes' theorem. Since $h_{i} \rightarrow \tilde{g}$ in $W^{1, p}$, applying Fubini's theorem we may select a subsequence (still denoted by $h_{i}$ ) such that

$$
\left.\left.h_{i}\right|_{\mathbb{S}^{n}(r)} \rightarrow \tilde{g}\right|_{\mathbb{S}^{n}(r)} \text { in } W^{1, p}\left(\mathbb{S}^{n}(r)\right) \text { for a.e. } 0<r<2 ;
$$

see [27, pp. 189-190]. Fix such $r$ in the interval $1<r<2$. Then Hölder's inequality yields

$$
\int_{\mathbb{B}^{n+1}(r)} h_{i}^{*}(d \omega) \rightarrow \int_{\mathbb{B}^{n+1}(r)} \tilde{g}^{*}(d \omega)=\int_{\mathbb{B}^{n+1}(1)} g^{*}(d \omega) .
$$

On the other hand, another application of Hölder's inequality yields

$$
\int_{\mathbb{B}^{n+1}(r)} h_{i}^{*}(d \omega)=\int_{\mathbb{S}^{n}(r)} h_{i}^{*} \omega \rightarrow \int_{\mathbb{S}^{n}(r)} \tilde{g}^{*} \omega=\int_{\mathbb{S}^{n}(1)} g^{*} \omega,
$$


where the last equality follows from the fact that $\tilde{g}$ on $\mathbb{S}^{n}(r)$ is the rescaling of $g$ on $\mathbb{S}^{n}(1)$. Hence

and the lemma follows.

$$
\int_{\mathbb{B}^{n+1}(1)} g^{*}(d \omega)=\int_{\mathbb{S}^{n}(1)} g^{*} \omega
$$

Now we complete the proof of Proposition 4.8. Let $\omega$ be the pullback by $\psi^{-1}$ of the volume form of $\mathbb{S}^{n}$ to $\psi\left(\mathbb{S}^{n}\right) \subset \mathbb{R}^{k}$. We can assume that $\omega$ is a smooth form. Indeed, we can extend it smoothly from $\psi\left(\mathbb{S}^{n}\right)$ to $\mathbb{R}^{k}$ using local extensions in coordinate systems and a partition of unity. The extended form may vanish outside a small neighborhood of $\psi\left(\mathbb{S}^{n}\right)$, but it does not matter. According to Lemma 4.9 we have

$$
\int_{\mathbb{B}^{n+1}} \tilde{\psi}^{*}(d \omega)=\int_{\mathbb{S}^{n}} \psi^{*} \omega=\mathcal{H}^{n}\left(\mathbb{S}^{n}\right)>0 .
$$

This implies that the rank of $d \tilde{\psi}$ is $n+1$ on a set of positive measure and hence

$$
\left|J_{\tilde{\psi}}\right|=\sqrt{\operatorname{det}(d \tilde{\psi})^{T}(d \tilde{\psi})}>0
$$

on a set of positive measure. According to the area formula [18]

$$
\int_{\tilde{\psi}\left(\mathbb{B}^{n+1}\right)} \mathcal{H}^{0}\left(\tilde{\psi}^{-1}(y)\right) d \mathcal{H}^{n+1}(y)=\int_{\mathbb{B}^{n+1}}\left|J_{\tilde{\psi}}\right|>0
$$

where $\mathcal{H}^{0}$ stands for the counting measure. This, however, implies that the set $\tilde{\psi}\left(\mathbb{B}^{n+1}\right)$ has positive $(n+1)$-dimensional measure. This completes the proof of Proposition 4.8

In view of the preceding discussion, the proof of Proposition 4.7 is complete.

Remark 4.10. A modification of the proof yields the following stronger version of Proposition 4.7. Let $\psi_{1}, \ldots, \psi_{N}: \mathbb{S}^{n} \hookrightarrow \mathbb{H}^{n}$ be smooth horizontal embeddings such that

$$
\psi_{i}\left(\mathbb{S}^{n}\right) \backslash \bigcup_{j \neq i} \psi_{j}\left(\mathbb{S}^{n}\right) \neq \emptyset \quad \text { for some } i=1, \ldots, N
$$

Then $\psi_{1}, \ldots, \psi_{N}$ are independent as elements of $\pi_{n}^{\mathrm{Lip}}\left(\mathbb{H}^{n}\right)$, i.e., no nontrivial relations among these embeddings exist. Indeed, suppose that some finite linear combination $\sum_{j} m_{j} \psi_{j}$ was equal to zero in $\pi_{n}^{\mathrm{Lip}}\left(\mathbb{H}^{n}\right)$. Since Lipschitz homotopy groups were defined via mappings from the pair $\left(Q^{n}, \partial Q^{n}\right)$, we interpret this assumption as providing a Lipschitz map $\tilde{\psi}$ from $Q^{n+1}$ to $\mathbb{H}^{n}$ which is constant on all but one face of $Q^{n+1}$ and which is given by the concatenation of $m_{1}$ copies of $\psi_{1}, m_{2}$ copies of $\psi_{2}, \ldots, m_{N}$ copies of $\psi_{N}$ on the remaining face. Let $\psi: \partial Q^{n+1} \rightarrow \mathbb{H}^{n}$ denote the restriction of $\tilde{\psi}$ to the boundary.

Fix $i$ so that (4.1) holds. Then $\psi_{i}^{-1}\left(\psi_{i}\left(\mathbb{S}^{n}\right) \backslash \bigcup_{j \neq i} \psi_{j}\left(\mathbb{S}^{n}\right)\right)$ has nonempty interior $V$ in $\mathbb{S}^{n}$ (since $\psi_{i}$ is an embedding). Let $\omega$ be the pullback by $\psi^{-1}$ of a smooth $n$-form supported in $V$ such that $\int_{V} \psi^{*} \omega>0$. The remainder of the proof proceeds as before.

Note, in particular, that if $N=2$, then the negation of (4.1) implies that $\psi_{1}\left(\mathbb{S}^{n}\right)=\psi_{2}\left(\mathbb{S}^{n}\right)$. Thus two embedded spheres are dependent as elements of $\pi_{n}^{\text {Lip }}\left(\mathbb{H}^{n}\right)$ if and only if their images coincide, in which case they agree up to a reparameterization of $\mathbb{S}^{n}$. 
Theorem 4.11. (1) If $1 \leq k<n$, then $\pi_{k}^{\text {Lip }}\left(\mathbb{H}^{n}\right)=0$.

(2) $\pi_{n}^{\text {Lip }}\left(\mathbb{H}^{n}\right)$ is uncountably generated for $n>0$.

Proof. First, we prove (1). Theorem 2.6 immediately implies that every Lipschitz map $f: \mathbb{S}^{k} \rightarrow \mathbb{H}^{n}$ admits a Lipschitz extension $\tilde{f}: \mathbb{B}^{k+1} \rightarrow \mathbb{H}^{n}$ provided $1 \leq k<n$. Hence $\pi_{k}^{\text {Lip }}\left(\mathbb{H}^{n}\right)=0$.

There exists a bi-Lipschitz embedding $\phi: \mathbb{S}^{n} \rightarrow \mathbb{H}^{n}$ (see Theorem 3.2). Proposition 4.7 guarantees that $\phi$ admits no Lipschitz extension $\tilde{\phi}: \mathbb{B}^{n+1} \rightarrow \mathbb{H}^{n}$. Hence $\pi_{n}^{\text {Lip }}\left(\mathbb{H}^{n}\right) \neq 0$. To see that $\pi_{n}^{\text {Lip }}\left(\mathbb{H}^{n}, x_{0}\right)$ has uncountably many generators we need to show that there are uncountably many Lipschitz mappings from $\mathbb{S}^{n}$ to $\mathbb{H}^{n}$ which map a fixed point on $\mathbb{S}^{n}$ to $x_{0}$ and whose homotopy classes in $\pi_{n}^{\mathrm{Lip}}\left(\mathbb{H}^{n}, x_{0}\right)$ have no nontrivial relations. Applying a translation if necessary, we may assume that

$$
\phi(-1,0, \ldots, 0)=x_{0}=(0, \ldots, 0) \in \mathbb{H}^{n} .
$$

An uncountable family of maps as above is then obtained by considering the maps $\phi_{r}: \mathbb{S}^{n} \rightarrow \mathbb{H}^{n}$ given by $\phi_{r}(x)=\delta_{r}(\phi(x))$ for $r>0$, where $\delta_{r}: \mathbb{H}^{n} \rightarrow \mathbb{H}^{n}$ is the dilation given in (2.2). The fact that there are no relations between these mappings follows from the discussion in Remark 4.10. This completes the proof of (2).

When $n>1$, we can also show that the higher homotopy groups of spheres arise as subgroups of the horizontal homotopy groups of $\mathbb{H}^{n}$.

Theorem 4.12. For $k \geq n \geq 2, \pi_{k}^{H}\left(\mathbb{H}^{n}\right)$ is nontrivial whenever $\pi_{k}\left(\mathbb{S}^{n}\right)$ is nontrivial.

We use the following lemma.

Lemma 4.13. Let $f: \mathbb{R}^{m} \rightarrow \mathbb{H}^{n}$ be smooth and horizontal. Then df has rank at most $n$.

Proof. Without loss of generality, assume $f$ is not constant. Suppose that rank $d f_{x_{0}}$ $=k$ is maximized for some $x_{0} \in \mathbb{R}^{m}$. Since $x \mapsto \operatorname{rank} d f_{x}$ is lower semicontinuous, $\operatorname{rank} d f_{x}=k$ for all $x$ in some neighborhood $U$ of $x_{0}$. We may choose a smooth $k$ dimensional submanifold $N \subset U$ so that $\left.d f\right|_{N}$ is injective and hence $\left.f\right|_{N}: N \rightarrow \mathbb{H}^{n}$ is a smooth immersion. Restricting to a submanifold $N^{\prime} \subset N$ if necessary, we obtain a smooth embedding $\left.f\right|_{N^{\prime}}: N^{\prime} \rightarrow \mathbb{H}^{n}$. Then $f\left(N^{\prime}\right)$ is a smooth embedded $k$-dimensional horizontal submanifold in $\mathbb{H}^{n}$, and so $k \leq n$.

Proof of Theorem 4.12, In the following argument we use the specific map $\phi$ constructed in Subsection 3.1] see (3.8) for a precise formula.

It is clear that the standard projection

$$
\mathbb{H}^{n} \cong \mathbb{C}^{n} \times \mathbb{R} \rightarrow \Re\left(\mathbb{C}^{n}\right) \times \mathbb{R} \cong \mathbb{R}^{n+1}
$$

is injective on the image of $\phi$. Furthermore, up to a diffeomorphism of $\mathbb{R}^{n+1}$ we may assume that $\pi \circ \phi\left(\mathbb{S}^{n}\right)$ is the unit sphere $\mathbb{S}^{n} \subset \mathbb{R}^{n+1}$.

Suppose that $f: \mathbb{S}^{k} \rightarrow \mathbb{S}^{n}$ is homotopically nontrivial but that $\phi \circ f$ can be extended to a smooth horizontal map $\tilde{f}: \mathbb{B}^{k+1} \rightarrow \mathbb{H}^{n}$. Projecting to $\mathbb{R}^{n+1}$ as in (4.2), write $\tilde{F}=\pi \circ \tilde{f}$.

By Sard's Theorem, $\tilde{F}$ has full rank at the preimage of a.e. point. Lemma 4.13 implies that the image of $\tilde{F}$ has measure zero, so there exists a point in $\mathbb{B}^{n+1}$ which is not in the image of $\tilde{F}$. Projecting radially from this point to $\mathbb{S}^{n}=\partial \mathbb{B}^{n+1}$, we get 
(up to the diffeomorphism $\pi \circ \phi$ ) an extension of $f$ to $B^{k+1}$. This contradicts the nontriviality of $f$.

The preceding argument is summarized in the following diagram:

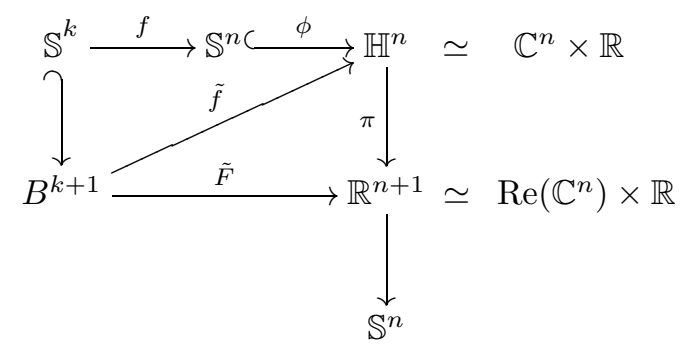

We conclude that $\phi \circ f$ represents a nontrivial element in $\pi_{k}^{\mathrm{H}}\left(\mathbb{H}^{n}\right)$ whenever $f$ represents a nontrivial element in $\pi_{k}\left(\mathbb{S}^{n}\right)$.

Remark 4.14. Every dilation of $\phi \circ f$ also represents a nontrivial element in $\pi_{k}^{\mathrm{H}}\left(\mathbb{H}^{n}\right)$, and all such elements are pairwise not Lipschitz homotopic. It follows that the horizontal homotopy groups of $\mathbb{H}^{n}$ are also uncountably infinitely generated for $k \geq n$ so that $\pi_{k}\left(\mathbb{S}^{n}\right)$ is nontrivial.

Allcock 2] proved a quadratic isoperimetric inequality in the symplectic space $\mathbb{R}^{2 n}, n \geq 2$. His result implies, in particular, that every closed loop enclosing zero symplectic area can be filled by an isotropic spanning disc. Lifting this result into the Heisenberg group $\mathbb{H}^{n}, n \geq 2$, and translating it into the language of Lipschitz homotopy groups leads to the conclusion

$$
\pi_{1}^{\mathrm{H}}\left(\mathbb{H}^{n}\right)=0 \quad \text { for } n \geq 2 .
$$

Compare Theorem 4.11 (1). The following question remains open.

Question 4.15. Is $\pi_{k}^{\mathrm{H}}\left(\mathbb{H}^{n}\right)=0$ for $1 \leq k<n$ and $n \geq 3$ ?

Question 4.16. Is $\pi_{k}^{\mathrm{H}}\left(\mathbb{H}^{1}\right)=0$ for $k>1$ ?

Question 4.17. Is the homomorphism $\pi_{k}\left(\mathbb{S}^{n}\right) \rightarrow \pi_{k}^{\text {Lip }}\left(\mathbb{H}^{n}\right)$ induced by a biLipschitz embedding $\phi: \mathbb{S}^{n} \rightarrow \mathbb{H}^{n}$ injective?

Remark 4.18. Following preparation of this paper, Wenger and Young [51, Theorem 1] surprisingly answered Question 4.17 in the negative for certain $k$ and $n$. More precisely, for $n+2 \leq k<2 n-1$, they proved that every composition of Lipschitz maps $\mathbb{S}^{k} \rightarrow \mathbb{S}^{n} \rightarrow \mathbb{H}^{n}$ extends to a Lipschitz map $\mathbb{B}^{k+1} \rightarrow \mathbb{H}^{n}$. Applying this result to any homotopically nontrivial map $\mathbb{S}^{k} \rightarrow \mathbb{S}^{n}$ composed with one of the bi-Lipschitz embeddings $\mathbb{S}^{n} \rightarrow \mathbb{H}^{n}$ discussed above yields the indicated counterexample to the result proposed in Question 4.17. Wenger and Young also proved (see [51, Corollary 4]) that $\pi_{k}^{\operatorname{Lip}}\left(\mathbb{H}^{1}\right)=0$ for $k \geq 2$.

For $n=2 d$, the preceding result of Wenger-Young is valid in the range $2 d+2 \leq$ $k \leq 4 d-2$. In 29], two of the authors (joint with Schikorra) employ a new version of the Hopf invariant for low rank Lipschitz maps to obtain nontriviality of the Lipschitz homotopy group $\pi_{4 d-1}^{\text {Lip }}\left(\mathbb{H}^{2 d}\right)$, with corresponding consequences for the Lipschitz nondensity in suitable Sobolev spaces. The paper 29] also contains an alternate proof of the nontriviality of $\pi_{n}^{\mathrm{Lip}}\left(\mathbb{H}^{n}\right)$. 


\section{Sobolev mappings into metric spaces}

Every separable metric space $(X, d)$ admits an isometric embedding into a Banach space. For example, given a dense subset $\left\{x_{i}\right\}_{i=1}^{\infty} \subset X$ and $x_{0} \in X$, the map

$$
\kappa: X \rightarrow \ell^{\infty}, \quad \kappa(x)=\left(d\left(x, x_{i}\right)-d\left(x_{0}, x_{i}\right)\right)_{i=1}^{\infty}
$$

is an example of such an isometric embedding. It is called the Kuratowski embedding. An important property of $\ell^{\infty}$ is that it is dual to a separable Banach space: $\ell^{\infty}=\left(\ell^{1}\right)^{*}$. Thus every separable metric space can be isometrically embedded into a Banach space which is dual to a separable Banach space.

Given an isometric embedding of $X$ into a Banach space $V$ (not necessarily dual to a separable Banach space), and an open set $\Omega \subset \mathbb{R}^{n}$, we define the Sobolev space of mappings $W^{1, p}(\Omega, X)$ as follows:

$$
W^{1, p}(\Omega, X)=\left\{f \in W^{1, p}(\Omega, V): f(x) \in X \text { a.e. }\right\} .
$$

The vector valued Sobolev space $W^{1, p}(\Omega, V)$ is a well-known object and can be defined using the notion of weak derivatives. This requires the notion of the Bochner integral.

If $V$ is any Banach space and $A \subset \mathbb{R}^{n}$ is (Lebesgue) measurable, we say that $f \in L^{p}(A, V)$ if

(1) $f$ is essentially separably valued: $f(A \backslash Z)$ is a separable subset of $V$ for some set $Z$ of Lebesgue measure zero,

(2) $f$ is weakly measurable: for every $v^{*} \in V^{*}$ with $\left\|v^{*}\right\| \leq 1,\left\langle v^{*}, f\right\rangle$ is measurable, and

(3) $\|f\| \in L^{p}(A)$.

If $f \in L^{1}(A, V)$ we define the integral

$$
\int_{A} f(x) d x
$$

as an element of $V$ in the Bochner sense; see [52, Chapter 5, Sections 4-5], 15]. The Bochner integral has two important properties:

$$
\left\langle v^{*}, \int_{A} f(x) d x\right\rangle=\int_{A}\left\langle v^{*}, f(x)\right\rangle d x
$$

for every $v \in V^{*}$, and

$$
\left\|\int_{A} f(x) d x\right\| \leq \int_{A}\|f(x)\| d x .
$$

We can now define the vector valued Sobolev space as follows. Let $\Omega \subset \mathbb{R}^{n}$ be an open set and $V$ any Banach space (not necessarily dual to a separable space). The Sobolev space $W^{1, p}(\Omega, V), 1 \leq p<\infty$, is defined as the class of all functions $f \in L^{p}(\Omega, V)$ such that for $i=1,2, \ldots, n$ there is $f_{i} \in L^{p}(\Omega, V)$ such that

$$
\int_{\Omega} \frac{\partial \varphi}{\partial x_{i}} f=-\int_{\Omega} \varphi f_{i}
$$

for every $\varphi \in C_{0}^{\infty}(\Omega)$, where the integrals are taken in the sense of Bochner. Note that the integrands are supported on compact subsets of $\Omega$. We denote $f_{i}=$ 
$\partial f / \partial x_{i}$ and call these functions weak partial derivatives of $f$. We also write $\nabla f=$ $\left(\partial f / \partial x_{1}, \ldots, \partial f / \partial x_{n}\right)$ and

$$
|\nabla f|=\left(\sum_{i=1}^{n}\left\|\frac{\partial f}{\partial x_{i}}\right\|^{2}\right)^{1 / 2} .
$$

Sometimes we will write $|\nabla f|_{V}$ to emphasize the Banach space with respect to which we compute the length of the gradient. The space $W^{1, p}(\Omega, V)$ is equipped with the norm

$$
\|f\|_{1, p}=\left(\int_{\Omega}\|f\|^{p}\right)^{1 / p}+\left(\int_{\Omega}|\nabla f|^{p}\right)^{1 / p} .
$$

It is easy to prove that $W^{1, p}(\Omega, V)$ is a Banach space. Using local coordinate systems we can also easily define the Sobolev space $W^{1, p}(M, V)$ and hence $W^{1, p}(M, X)$ for any compact Riemannian manifold $M$.

Let $\kappa:\left(\mathbb{H}^{n}, d_{c c}\right) \rightarrow \ell^{\infty}$ be the Kuratowski embedding used to define the space $W^{1, p}\left(M, \mathbb{H}^{n}\right)$. Then by definition $f \in W^{1, p}\left(M, \mathbb{H}^{n}\right)$ if and only if $\bar{f}=\kappa \circ f \in$ $W^{1, p}\left(M, \ell^{\infty}\right)$ and

$$
\|f\|_{1, p}=\left(\int_{M}\|\bar{f}\|_{\infty}^{p}\right)^{1 / p}+\left(\int_{M}|\nabla \bar{f}|_{\ell \infty}^{p}\right)^{1 / p} .
$$

If $M=\Omega \subset \mathbb{R}^{m}$ is a bounded domain and $f \in W^{1, p}\left(\Omega, \mathbb{H}^{n}\right)$, then

$$
\|f\|_{1, p}=\left(\int_{\Omega}\|\bar{f}\|_{\infty}^{p}\right)^{1 / p}+\left(\int_{\Omega}|\nabla f|_{\mathbb{H}}^{p}\right)^{1 / p} ;
$$

see Proposition 6.6

While the Sobolev space $W^{1, p}(M, V)$ can be defined for any Banach space $V$, it has particularly nice properties if $V$ is dual to a separable Banach space. Namely one can prove the following result. See e.g. 30.

Proposition 5.1. Let $\Omega \subset \mathbb{R}^{n}$ be an open set and let $V$ be dual to a separable Banach space $Y$. Then $f \in W^{1, p}(\Omega, V)$ if and only if $f \in L^{p}(\Omega, V)$ and the following two conditions hold:

(i) for every $v^{*} \in V^{*}$, we have $\left\langle v^{*}, f\right\rangle \in W^{1, p}(\Omega)$, and

(ii) there is a nonnegative function $g \in L^{p}(\Omega)$ such that

$$
\left|\nabla\left\langle v^{*}, f\right\rangle\right| \leq g \quad \text { a.e. }
$$

for every $v^{*} \in V^{*}$ with $\left\|v^{*}\right\| \leq 1$.

Moreover,

$$
\|f\|_{p}+\inf \|g\|_{p} \leq\|f\|_{1, p} \leq\|f\|_{p}+\sqrt{n} \inf \|g\|_{p},
$$

where the infimum is over the class of all $g$ that satisfy (5.4).

This result easily leads to the following characterization. See [34, Theorem 3.17] and 30 .

Proposition 5.2. Let $X$ be a separable metric space that is isometrically embedded into a Banach space that is dual to a separable Banach space. Let $M$ be a compact Riemannian manifold and $1 \leq p<\infty$. Then $f$ is in $W^{1, p}(M, X)$ if and only if $d\left(x_{0}, f\right) \in W^{1, p}(M)$ for every $x_{0} \in X$ and there is a nonnegative function $g \in$ $L^{p}(M)$ such that $\left|\nabla d\left(x_{0}, f\right)\right| \leq g$ a.e. for each $x_{0}$. 
The Sobolev spaces $W^{1, p}(M, X)$ behave well under Lipschitz postcomposition. See Reshetnyak [46, Corollary 1, p. 580] for the following result in the case when $M=\Omega$ is a Euclidean domain. The general statement given here is similar.

Proposition 5.3. Let $X$ and $Y$ be separable metric spaces that are isometrically embedded into Banach spaces that are dual to separable Banach spaces. Let $M$ be a compact Riemannian manifold and $1 \leq p<\infty$. If $F: X \rightarrow Y$ is Lipschitz, then the operation $f \mapsto F \circ f$ sends $W^{1, p}(M, X)$ into $W^{1, p}(M, Y)$. Furthermore, if $F$ is $L$-Lipschitz, then $|\nabla(F \circ f)| \leq L|\nabla f|$ a.e. in $\Omega$.

If $X$ is a separable metric space that is isometrically embedded into a Banach space $V$ that is dual to a separable Banach space, then $W^{1, p}(M, X) \subset W^{1, p}(M, V)$. It turns out that $W^{1, p}(M, X)$ mappings can be approximated by $\operatorname{Lip}(M, V)$ mappings, but the real question is whether they can be approximated by $\operatorname{Lip}(M, X)$ mappings.

Proposition 5.4 ([26]). If a Banach space $V$ is dual to a separable Banach space, $M$ is a compact Riemannian manifold, possibly with boundary, and $f \in W^{1, p}(M, V)$, $1 \leq p<\infty$, then for every $\epsilon>0$ there is $g \in \operatorname{Lip}(M, V)$ such that $\mid\{x: f(x) \neq$ $g(x)\} \mid<\epsilon$ and $\|f-g\|_{1, p}<\epsilon$.

The proof of this result is similar to the proof of Lemma 13 in [25], so it was stated in [26] without proof. On the other hand, there are some technical differences and since later on we will need arguments used in the proof, we decided to provide details.

Denote the volume measure on $M$ by $\mu$. For $h \in L^{1}(\mu)$ we define the HardyLittlewood maximal operator by

$$
\mathcal{M} h(x)=\sup _{r>0} \frac{1}{\mu(\mathbb{B}(x, r))} \int_{\mathbb{B}(x, r)}|h| d \mu .
$$

The following result is well known; see [1], [23], [28, Theorems 3.2, 3.3].

Lemma 5.5. Given a compact Riemannian manifold $M$, possibly with boundary, and $1 \leq p<\infty$, there is a constant $C=C(M, p)$ such that

$$
|u(x)-u(y)| \leq C d(x, y)(\mathcal{M}|\nabla u|(x)+\mathcal{M}|\nabla u|(y)) \quad \text { a.e. } x, y \in M
$$

for all $u \in W^{1, p}(M)$.

More precisely, there is a set $F \subset M$ of measure zero such that (5.5) holds for all $x, y \in M \backslash F$.

Let $V=Y^{*}$, where $Y$ is a separable Banach space and let $u \in W^{1, p}(M, V)$. For every $v^{*} \in Y \subset Y^{* *}=V^{*}$ with $\left\|v^{*}\right\|_{Y} \leq 1, x \mapsto\left\langle v^{*}, u(x)\right\rangle \in W^{1, p}(M)$ and $\left|\nabla\left\langle v^{*}, u\right\rangle\right| \leq|\nabla u|$. Hence

$$
\left|\left\langle v^{*}, u(x)-u(y)\right\rangle\right| \leq C d(x, y)(\mathcal{M}|\nabla u|(x)+\mathcal{M}|\nabla u|(u)) \quad \text { a.e. } x, y \text {. }
$$

Note that the implicit exceptional set in (5.6) depends a priori on $v^{*}$. However, let $D \subset Y$ be a countable and dense subset of the unit ball in $Y$. Since (5.6) holds a.e. for every $v^{*} \in D$ and $D$ is countable, there is a set $F \subset M$ of measure zero such that (5.6) holds for all $v^{*} \in D$ and all $x, y \in M \backslash F$. Then

$$
\|u(x)-u(y)\|=\sup _{v^{*} \in D}\left|\left\langle v^{*}, u(x)-u(y)\right\rangle\right| \leq C d(x, y)(\mathcal{M}|\nabla u|(x)+\mathcal{M}|\nabla u|(y))
$$

for all such $x, y$ and in particular, for a.e. $x, y$.

We proved the following result. See also 34 . 
Lemma 5.6. Let $M$ and $V$ be as in Proposition 5.4 and let $1 \leq p<\infty$. Then there is a constant $C=C(M, p)$ such that if $u \in W^{1, p}(M, V)$, then

$$
\|u(x)-u(y)\| \leq C d(x, y)(\mathcal{M}|\nabla u|(x)+\mathcal{M}|\nabla u|(y)) \quad \text { a.e. } x, y .
$$

We will also need the following fact, which is Lemma 3.1 in [26].

Lemma 5.7. Let $M$ and $V$ be as in Proposition [5.4 and $1 \leq p<\infty$. If $u_{1}, u_{2} \in$ $W^{1, p}(M, V)$ and $u_{1}=u_{2}$ on a measurable set $E \subset M$, then $\nabla u_{1}=\nabla u_{2}$ a.e. on $E$.

Proof of Proposition 5.4. Let $f \in W^{1, p}(M, V)$. We want to prove that $f$ can be approximated by Lipschitz mappings as stated in Proposition 5.4. Recall that

$$
\|f(x)-f(y)\| \leq C d(x, y)(\mathcal{M}|\nabla f|(x)+\mathcal{M}|\nabla f|(y)) \quad \text { a.e. } x, y
$$

by Lemma 5.6. Let

$$
E_{t}=\{x \in M: \mathcal{M}|\nabla f|(x) \leq t\} .
$$

The set $E_{t}$ is compact and weak type estimates for the maximal operator $\mathcal{M}$ give

$$
t^{p} \mu\left(M \backslash E_{t}\right) \rightarrow 0 \quad \text { as } t \rightarrow \infty .
$$

Note that the mapping

$$
\left.f\right|_{E_{t}}: E_{t} \rightarrow V
$$

is Lipschitz continuous with Lipschitz constant $2 C t$. Hence the function $\left.f\right|_{E_{t}}$ admits a $C^{\prime} t$-Lipschitz extension $f_{t}: M \rightarrow V$. Indeed, there is a Whitney decomposition of $M \backslash E_{t}$ into balls and subordinated Lipschitz partition of unity. This is to say there is a constant $C \geq 1$ depending on the Riemannian structure of $M$ only and a sequence $\left\{x_{i}\right\}_{i \in I}$ of points in $M \backslash E_{t}$ such that with $r_{i}=\operatorname{dist}\left(x_{i}, E_{t}\right) / 10$ we have

(a) $\bigcup_{i \in I} B\left(x_{i}, r_{i}\right)=M \backslash E_{t}$;

(b) $B\left(x_{i}, 5 r_{i}\right) \subset M \backslash E_{t}$ for all $i \in I$;

(c) for every $i \in I$ and all $x \in B\left(x_{i}, 5 r_{i}\right)$ we have $5 r_{i} \leq \operatorname{dist}\left(x, E_{t}\right) \leq 15 r_{i}$;

(d) no point of $M \backslash E_{t}$ belongs to more than $C$ balls $\left\{B\left(x_{i}, 5 r_{i}\right)\right\}_{i \in I}$;

(e) there is a family of Lipschitz continuous functions $\left\{\varphi_{i}\right\}_{i \in I}$ such that

$$
\operatorname{supp} \varphi_{i} \subset B\left(x_{i}, 2 r_{i}\right), 0 \leq \varphi_{i} \leq 1, \sum_{i \in I} \varphi_{i}=1
$$

and the Lipschitz constant of $\varphi_{i}$ is bounded by $C r_{i}^{-1}$.

Now it is a routine verification to show that the function

$$
f_{t}(x)=\left\{\begin{array}{cl}
f(x) & \text { if } x \in E_{t}, \\
\sum_{i \in I}\left(\frac{1}{\mu\left(B_{i}\right)} \int_{B_{i}} f d \mu\right) \varphi_{i}(x) & \text { if } x \notin E_{t},
\end{array}\right.
$$

is the desired $C^{\prime} t$-Lipschitz extension of $\left.f\right|_{E_{t}}$. For details, see for example [24, pp. 446-448], where the argument is presented in a slightly different, but related setting.

Observe that if $t \geq 1$ is sufficiently large, then the norm of $f_{t}$ is bounded by $C t$. Indeed, let $x_{0} \in E_{1}$. Then for any $x \in M$ we have

$$
\left\|f_{t}(x)\right\| \leq\left\|f_{t}(x)-f_{t}\left(x_{0}\right)\right\|+\left\|f\left(x_{0}\right) \mid\right\| \leq C t d\left(x, x_{0}\right)+\left\|f\left(x_{0}\right)\right\|
$$


and it suffices to observe that $d\left(x, x_{0}\right) \leq \operatorname{diam} M$ and take $t \geq\left\|f\left(x_{0}\right)\right\|$. Thus

$$
\begin{aligned}
\left(\int_{M}\left\|f-f_{t}\right\|^{p}\right)^{1 / p} & \leq\left(\int_{M \backslash E_{t}}\|f\|^{p}\right)^{1 / p}+\left(\int_{M \backslash E_{t}}\left\|f_{t}\right\|^{p}\right)^{1 / p} \\
& \leq\left(\int_{M \backslash E_{t}}\|f\|^{p}\right)^{1 / p}+C\left(t^{p} \mu\left(M \backslash E_{t}\right)\right)^{1 / p} \rightarrow 0
\end{aligned}
$$

as $t \rightarrow \infty$. We applied here the weak type estimate (5.7). For derivatives we have a similar estimate

$$
\left(\int_{M}\left|\nabla\left(f-f_{t}\right)\right|^{p}\right)^{1 / p} \leq\left(\int_{M \backslash E_{t}}|\nabla f|^{p}\right)^{1 / p}+C\left(t^{p} \mu\left(M \backslash E_{t}\right)\right)^{1 / p} \rightarrow 0
$$

as $t \rightarrow \infty$. Here we applied Lemma 5.7 and the weak type estimate (5.7). This completes the proof.

\section{SOBOLEV CONVERGENCE FOR $\mathbb{H}^{n}$-VALUED MAPS}

Theorem 1.7 is analogous to a similar phenomenon which holds in the Euclidean context. See [26]. Its proof is based on an estimate relating the weak ${ }^{*}$ derivative of the difference between two Sobolev maps, postcomposed with the Kuratowski embedding, to the norm of the gradients of the individual mappings.

We begin by recalling the notion of weak $^{*}$ derivative.

Definition 6.1. Let $V$ be a Banach space which is dual to a separable Banach space. Let $f:[a, b] \rightarrow V$. A vector $v \in V$ is called a weak ${ }^{*}$ derivative of $f$ at $s \in[a, b]$ if $v$ is the weak ${ }^{*}$ limit of the vectors $h^{-1}(f(s+h)-f(s))$ as $h \rightarrow 0$.

For the following lemma, see Lemmas 2.8 and 2.13 of [30]. See also Lemma 2.3 in $[26$.

Lemma 6.2. Let $V$ be a Banach space which is dual to a separable Banach space $Y$ and let $f:[a, b] \rightarrow V$ be absolutely continuous. Then the limit

$$
g(s):=\lim _{h \rightarrow 0} h^{-1}\|f(s+h)-f(s)\|
$$

exists for a.e. $s$, and $g \in L^{1}([a, b])$. Furthermore, for a.e. $s \in[a, b]$ there exists a unique weak ${ }^{*}$ derivative $f^{\prime}(s)$ of $f$ at $s$, and $\left\|f^{\prime}(s)\right\| \leq g(s)$.

In the case when $V=\ell^{\infty}$, the weak* derivative can be computed explicitly. If $f=\left(f^{i}\right)_{i=1}^{\infty}$ is absolutely continuous from $[a, b]$ to $\ell^{\infty}$, then

$$
f^{\prime}(s)=\left(\left(f^{i}\right)^{\prime}(s)\right)_{i=1}^{\infty}
$$

whenever $f^{\prime}(s)$ is defined. See, for example, 26 .

Let $\left(p_{i}\right)_{i=1}^{\infty}$ be a dense subset of $\mathbb{H}^{n}, p_{0} \in \mathbb{H}^{n}$ and let $\kappa:\left(\mathbb{H}^{n}, d_{c c}\right) \rightarrow \ell^{\infty}$ be the Kuratowski embedding given by

$$
\kappa(p)=\left(d_{c c}\left(p, p_{i}\right)-d_{c c}\left(p_{0}, p_{i}\right)\right)_{i=1}^{\infty} .
$$

If $f:[a, b] \rightarrow \mathbb{H}^{n}$ is absolutely continuous, then $\bar{f}=\kappa \circ f:[a, b] \rightarrow \ell^{\infty}$ is absolutely continuous and

$$
\bar{f}^{\prime}(s)=\left(\frac{d}{d s} d_{c c}\left(f(s), p_{i}\right)\right)_{i=1}^{\infty} \quad \text { a.e. }
$$


The following lemma is a variation on Lemma 2.6 in [26].

Lemma 6.3. Let $f, g:[a, b] \rightarrow \mathbb{H}^{n}$ be absolutely continuous, and let $\kappa:\left(\mathbb{H}^{n}, d_{c c}\right) \rightarrow$ $\ell^{\infty}$ be the Kuratowski embedding. Then $\bar{f}=\kappa \circ f$ and $\bar{g}=\kappa \circ g$ are absolutely continuous, and the weak $k^{*}$ derivative $(\bar{f}-\bar{g})^{\prime}:[a, b] \rightarrow \ell^{\infty}$ satisfies

$$
\left\|(\bar{f}-\bar{g})^{\prime}(s)\right\|_{\infty} \geq \max \left\{\left|f^{\prime}(s)\right|_{\mathbb{H}},\left|g^{\prime}(s)\right|_{\mathbb{H}}\right\} \geq \frac{1}{2}\left(\left|f^{\prime}(s)\right|_{\mathbb{H}}+\left|g^{\prime}(s)\right|_{\mathbb{H}}\right)
$$

for almost every $s \in[a, b]$ such that $f(s)^{-1} * g(s) \notin Z$.

Note that $f^{\prime}(s)$ is horizontal for a.e. $s$ whenever $f:[a, b] \rightarrow \mathbb{H}^{n}$ is absolutely continuous.

Proof. It is easy to see that

$$
(\bar{f}-\bar{g})^{\prime}(s)=\left(\frac{d}{d s} d_{c c}\left(f(s), p_{i}\right)-\frac{d}{d s} d_{c c}\left(g(s), p_{i}\right)\right)_{i=1}^{\infty}
$$

for a.e. $s \in[a, b]$.

Let $S$ be the set of points $s \in[a, b]$ so that $f(s)^{-1} * g(s) \notin Z$. Fix $s \in S$ so that both $f$ and $g$ are differentiable at $s$ and (6.2) holds. Almost every $s \in S$ is of this type. If $f^{\prime}(s)=g^{\prime}(s)=0$, the inequality is obvious. Assume then, without loss of generality, that $f^{\prime}(s) \neq 0$ and $\left|f^{\prime}(s)\right|_{\mathbb{H}} \geq\left|g^{\prime}(s)\right|_{\mathbb{H}}$.

An application of Lemma 2.1, or more specifically, of (2.7), yields that

$$
\frac{d}{d s} d_{c c}\left(f(s), p_{i}\right)=\left\langle\nabla_{\mathbb{H}} d_{p_{i}}(f(s)), f^{\prime}(s)\right\rangle_{\mathbb{H}}
$$

whenever $i$ is such that $p_{i}^{-1} * f(s) \notin Z$.

If $L_{\sigma} g=\sigma * g$ is the left translation on $\mathbb{H}^{n}$, then $d L_{-f(s)}(f(s)): H_{f(s)} \mathbb{H}^{n} \rightarrow$ $H_{o} \mathbb{H}^{n}$. Hence

$$
t \mapsto \exp \left(t d L_{-f(s)}(f(s)) f^{\prime}(s)\right)
$$

is a geodesic passing through $o$ at $t=0$. Here exp denotes the exponential map from the Lie algebra of $\mathbb{H}^{n}$ to the group itself. We are also using the fact that exp is the identity map, [19, p. 3] and that straight lines passing through $o$ and contained in $\mathbb{C}^{n} \times\{0\} \subset \mathbb{C}^{n} \times \mathbb{R}=\mathbb{H}^{n}$ are geodesics, [40, Theorem 1]. Thus

$$
\gamma(t)=f(s) * \exp \left(t d L_{-f(s)}(f(s)) f^{\prime}(s)\right)
$$

is a geodesic such that $\gamma(0)=f(s)$ and $\gamma^{\prime}(0)=f^{\prime}(s)$. Given $\delta>0$, it follows from Remark 2.3 that

$$
-\left|f^{\prime}(s)\right|_{\mathbb{H}}=\left.\frac{d}{d t}\right|_{t=0} d_{c c}(\gamma(t), \gamma(\delta))=\left\langle\nabla_{\mathbb{H}} d_{\gamma(\delta)}(f(s)), f^{\prime}(s)\right\rangle_{\mathbb{H}}=\frac{d}{d s} d_{c c}(f(s), \gamma(\delta)) .
$$

Given $\epsilon>0$ and $\delta>0$ it follows from the continuity of the derivative of $d_{q}$ that we can find $p_{i}$ sufficiently close to $\gamma(\delta)$ so that

$$
\frac{d}{d s} d_{c c}\left(f(s), p_{i}\right) \leq-\left|f^{\prime}(s)\right|_{\mathbb{H}}+\epsilon .
$$

Similarly, we can find $p_{j}$ sufficiently close to $\gamma(-\delta)$ so that

$$
\frac{d}{d s} d_{c c}\left(f(s), p_{j}\right) \geq\left|f^{\prime}(s)\right|_{\mathbb{H}}-\epsilon .
$$

Since $d_{c c}$ is $C^{\infty}$ away from the center $Z$ (Lemma 2.1) and $f(s)^{-1} * g(s) \notin Z$, we may choose $p_{i}$ and $p_{j}$ so close to $f(s)$ that

$$
\left|\frac{d}{d s} d_{c c}\left(g(s), p_{i}\right)-\frac{d}{d s} d_{c c}\left(g(s), p_{j}\right)\right|<\epsilon .
$$

Hence one of the following quantities is greater than or equal to $\left|f^{\prime}(s)\right|_{\mathbb{H}}-2 \epsilon$ :

$$
\left|\frac{d}{d s} d_{c c}\left(f(s), p_{i}\right)-\frac{d}{d s} d_{c c}\left(g(s), p_{i}\right)\right|
$$


or

$$
\left|\frac{d}{d s} d_{c c}\left(f(s), p_{j}\right)-\frac{d}{d s} d_{c c}\left(g(s), p_{j}\right)\right|
$$

Letting $\epsilon \rightarrow 0$ gives

$$
\left\|(\bar{f}-\bar{g})^{\prime}(s)\right\|_{\infty} \geq\left|f^{\prime}(s)\right|_{\mathbb{H}}=\max \left\{\left|f^{\prime}(s)\right|_{\mathbb{H}},\left|g^{\prime}(s)\right|_{\mathbb{H}}\right\} .
$$

This completes the proof of the lemma.

Our next task is to replace the one-dimensional source space in Lemma 6.3 with a domain in a higher-dimensional Euclidean space.

Lemma 6.4. Let $\Omega$ be a bounded domain in $\mathbb{R}^{m}$, let $f, g \in W^{1,1}\left(\Omega, \mathbb{H}^{n}\right)$ and let $\kappa:\left(\mathbb{H}^{n}, d_{c c}\right) \rightarrow \ell^{\infty}$ be the Kuratowski embedding. Then $\bar{f}=\kappa \circ f$ and $\bar{g}=\kappa \circ g$ are in $W^{1,1}\left(\Omega, \ell^{\infty}\right)$ and

$$
|\nabla(\bar{f}-\bar{g})|_{\ell^{\infty}} \geq \frac{1}{4}\left(|\nabla f|_{\mathbb{H}}+|\nabla g|_{\mathbb{H}}\right) \chi_{\{f-g \notin Z\}} \quad \text { a.e. }
$$

Proof. This follows from absolute continuity along almost all lines parallel to the coordinate axes, the definition of the weak ${ }^{*}$ derivative and Lemma 6.3.

In order to take advantage of this inequality, we need to take care of the set where the functions $f$ and $g$ differ by a point in the center $Z$. The following lemma accomplishes this.

Lemma 6.5. Let $\Omega, f, g$ be as in Lemma 6.4 and let $S$ be the set of points $p \in \Omega$ for which $f(p)-g(p) \in Z$. Then $\nabla f=\nabla g$ almost everywhere in $S$.

Proof. First, we consider the case $m=1$. Assume that $\Omega=[a, b]$ and $f, g:[a, b] \rightarrow$ $\mathbb{H}^{n}$ are absolutely continuous. Let $p \in S$ and suppose that $f^{\prime}(p)$ and $g^{\prime}(p)$ exist and are horizontal, but are not equal. Then

$$
(f(p+\epsilon)-g(p+\epsilon))-(f(p)-g(p))=\epsilon\left(f^{\prime}(p)-g^{\prime}(p)\right)+o(\epsilon) .
$$

Since $f^{\prime}(p)$ and $g^{\prime}(p)$ are both horizontal, their difference cannot lie in $Z$. Hence for $\epsilon$ sufficiently small, $f(p+\epsilon)-g(p+\epsilon) \notin Z$, because otherwise the left-hand side of (6.6) would belong to $Z$ while the right-hand side would not. It follows that $p$ is an isolated point of $S$. The set $E$ of such points is at most countable.

Now for the general case. Assume that $f$ and $g$ are bounded $W^{1,1}$ maps from $\Omega \subset \mathbb{R}^{m}$ to $\mathbb{H}^{n}$. Let $E$ denote the set of $p \in S$ so that $\nabla f(p)$ and $\nabla g(p)$ exist but are not equal. For $p \in E$, there exists $i \in\{1, \ldots, m\}$ so that $\frac{\partial}{\partial x_{i}} f(p)$ and $\frac{\partial}{\partial x_{i}} g(p)$ are not equal. Applying the argument from the previous paragraph, we find a nontrivial line segment $L$ parallel to the $e_{i}$ axis and passing through $p$, so that $L \cap S=\{p\}$. Denote the set of such points by $E_{i}$. Then $E=E_{1} \cup \cdots \cup E_{m}$. Repeated application of Fubini's theorem shows that each of the sets $E_{i}$ has Lebesgue $m$-measure zero in $\Omega$. Hence the measure of $E$ is equal to zero.

Proposition 6.6. Let $\Omega$ be a domain in $\mathbb{R}^{m}$, let $f \in W^{1,1}\left(\Omega, \mathbb{H}^{n}\right)$ and let $\kappa$ : $\left(\mathbb{H}^{n}, d_{c c}\right) \rightarrow \ell^{\infty}$ be the Kuratowski embedding. Then $\bar{f}=\kappa \circ f \in W^{1,1}\left(\Omega, \ell^{\infty}\right)$ and

$$
|\nabla \bar{f}|_{\ell^{\infty}}=|\nabla f|_{\mathbb{H}} \quad \text { a.e. }
$$

where $|\nabla f|_{\mathbb{H}}$ was defined in (1.2) and on the left-hand side of the inequality we have the norm defined in (5.3). 
Proof. First we consider the case $m=1$. Assume that $f:[a, b] \rightarrow \mathbb{H}^{n}$ is absolutely continuous. Then at a point where $\bar{f}^{\prime}(s)$ exists we have

$$
\left\|\bar{f}^{\prime}(s)\right\|_{\infty}=\sup _{i}\left|\frac{d}{d s} d_{c c}\left(f(s), p_{i}\right)\right| .
$$

Since

$$
\begin{aligned}
\left|\frac{d}{d s} d_{c c}\left(f(s), p_{i}\right)\right| & \leq \liminf _{h \rightarrow 0} \frac{d_{c c}(f(s+h), f(s))}{|h|} \\
& \leq \liminf _{h \rightarrow 0} \frac{1}{h} \int_{s}^{s+h}\left|f^{\prime}(\tau)\right|_{\mathbb{H}} d \tau=\left|f^{\prime}(s)\right|_{\mathbb{H}}
\end{aligned}
$$

for a.e. $s \in[a, b]$ we have $\left\|\bar{f}^{\prime}(s)\right\|_{\infty} \leq\left|f^{\prime}(s)\right|_{\mathbb{H}}$. On the other hand, inequality (6.4) implies that $\left\|\bar{f}^{\prime}(s)\right\|_{\infty} \geq\left|f^{\prime}(s)\right|_{\mathbb{H}}$. Hence $\left\|\bar{f}^{\prime}(x)\right\|_{\infty}=\left|f^{\prime}(s)\right|_{\mathbb{H}}$ a.e. The general case $m \geq 1$ follows from the case $m=1$ and absolute continuity of $f$ along almost all lines parallel to the coordinate axes.

Proof of Theorem 1.7. Suppose that $\Omega$ and the maps $f_{k}, f$ are as in the statement of the theorem, and assume that $f_{k}$ converges to $f$ in $W^{1, p}\left(\Omega, \mathbb{H}^{n}\right)$. Using (6.5) we find

$$
\int_{\left\{f_{k}-f \notin Z\right\}}\left(\left|\nabla f_{k}\right|_{\mathbb{H}}+|\nabla f|_{\mathbb{H}}\right)^{p} \leq C \int_{\Omega}\left|\nabla\left(\bar{f}_{k}-\bar{f}\right)\right|_{\ell^{\infty}}^{p} \rightarrow 0
$$

as $k \rightarrow \infty$. This completes the proof.

Proof of Corollary 1.8, By working with charts we may assume that $M=\Omega$ is a bounded domain in $\mathbb{R}^{m}$. Assume that $f_{k}$ converges to $f$ in $W^{1, p}\left(\Omega, \mathbb{H}^{n}\right)$. Clearly, $f_{k} \rightarrow f$ in $L^{p}\left(\Omega, \mathbb{R}^{2 n+1}\right)$. For each $k$, let $S_{k}:=\left\{p \in \Omega: f(p)-f_{k}(p) \in Z\right\}$. By Theorem 1.7

$$
\int_{\Omega \backslash S_{k}}\left|\nabla f_{k}\right|^{p}+|\nabla f|^{p} \leq C \int_{\Omega \backslash S_{k}}\left|\nabla f_{k}\right|_{\mathbb{H}}^{p}+|\nabla f|_{\mathbb{H}}^{p} \rightarrow 0 .
$$

The inequality follows from the fact that the ranges of all mappings $f_{k}, f$ are contained in a common bounded subset of $\mathbb{H}^{n}$ and the Heisenberg norm $|v|_{\mathbb{H}}$ and the Euclidean norm $|v|$ are comparable for horizontal vectors $v \in H_{p} \mathbb{H}^{n}$ when $p$ varies over a bounded set in $\mathbb{H}^{n}$. By Lemma 6.5 we have $\int_{S_{k}}\left|\nabla\left(f_{k}-f\right)\right|^{p}=0$. Note that here we refer to the Euclidean difference and the Euclidean norm on $\nabla\left(f_{k}-f\right)$. Combining these two statements completes the proof.

Remark 6.7. Everything discussed in this section works also for the Korányi metric $d_{K}$ (see (2.4) for the definition). The proofs are easier, since $d_{K}$ is clearly smooth on the set $\left\{(p, q) \in \mathbb{H}^{n} \times \mathbb{H}^{n}: p \neq q\right\}$. In particular, there is no need to make any special distinction for pairs of points $p, q \in \mathbb{H}^{n}$ with $p \neq q$ but $q^{-1} * p \in Z$.

6.1. The definition of Capogna and Lin. We conclude this section by discussing the equivalence of the above definition for the Sobolev space $W^{1, p}\left(M, \mathbb{H}^{n}\right)$ with that of Capogna and Lin. This equivalence is already known and can be deduced, for instance, by passing through the definition of Korevaar and Schoen. Nevertheless, we find it instructive to give a self-contained, direct discussion. In order to avoid issues with the integrability of the function itself, we restrict attention to bounded functions. Again by working in charts we may assume that $M=\Omega$ is a Euclidean domain. 
To prove the equivalence with the definition of Capogna and Lin it suffices to prove the following result, because the condition stated in the result is equivalent to their definition of the Heisenberg valued Sobolev space. See Theorems 2.11 and 2.15 in 11 .

Proposition 6.8. A bounded function $f=(z, t)=\left(x_{1}, y_{1}, \ldots, x_{n}, y_{n}, t\right): \Omega \rightarrow$ $\mathbb{H}^{n}$ lies in $W^{1, p}\left(\Omega, \mathbb{H}^{n}\right)$ if and only if $f$ is an element of the usual Sobolev space $W^{1, p}\left(\Omega, \mathbb{R}^{2 n+1}\right)$ and satisfies the contact equation

$$
\nabla t=2 \sum_{j=1}^{n}\left(y_{j} \nabla x_{j}-x_{j} \nabla y_{j}\right)
$$

almost everywhere in $\Omega$.

Proof. To prove the "only if" statement, we consider a bounded function $f \in$ $W^{1, p}\left(\Omega, \mathbb{H}^{n}\right)$. Combining the fact that the identity map from $\mathbb{H}^{n}$ to $\mathbb{R}^{2 n+1}$ is Lipschitz on bounded sets with Proposition 5.3 shows that $f \in W^{1, p}\left(\Omega, \mathbb{R}^{2 n+1}\right)$. Moreover, the ACL property of $f$ (see, for instance, the proof of Theorem 2.2 in 27] ) yields that the image of almost every line parallel to a coordinate axis in $\Omega$, is a horizontal curve. Consequently, the contact equation (6.7) is satisfied a.e. on such lines. By Fubini's theorem, (6.7) holds a.e. in $\Omega$.

To prove the "if" statement we reverse the argument. Consider a bounded function $f \in W^{1, p}\left(\Omega, \mathbb{R}^{2 n+1}\right)$ which satisfies (6.7) almost everywhere. We first note that $f$ is ACL as a map from $\Omega$ to $\mathbb{R}^{2 n+1}$. From (6.7), by an application of Fubini's theorem, we conclude that the image of almost every line parallel to a coordinate axis in $\Omega$ is a horizontal curve. Along horizontal curves in $\mathbb{H}^{n}$, the Hausdorff 1-measures computed with respect to the Euclidean and Carnot-Carathéodory metrics are mutually absolutely continuous. Hence $f$ is ACL as a map to $\mathbb{H}^{n}$ and so also as a map to $\kappa\left(\mathbb{H}^{n}\right) \subset \ell^{\infty}$. It now follows that the weak ${ }^{*}$ partial derivatives of $f$ exist almost everywhere and are bounded above (almost everywhere) by an $L^{p}$ function. By Proposition [5.1, $f$ lies in $W^{1, p}\left(\Omega, \mathbb{H}^{n}\right)$.

\section{Density And nONDENSity OF Lipschitz Maps In $W^{1, p}\left(M, \mathbb{H}^{n}\right)$}

7.1. Proof of Proposition 1.3. Let $\psi: \mathbb{S}^{n} \rightarrow \mathbb{H}^{n}$ be a bi-Lipschitz embedding whose existence was established in Theorem 1.6. We can also assume that $\psi$ is a $C^{\infty}$ embedding as a map from $\mathbb{S}^{n}$ to $\mathbb{R}^{2 n+1}$. Let

$$
f(x)=\psi\left(\frac{x}{|x|}\right) \in W^{1, p}\left(\mathbb{B}^{n+1}, \mathbb{H}^{n}\right), \quad 1 \leq p<n+1 .
$$

We will prove that the mapping $f$ cannot be approximated by Lipschitz mappings $\operatorname{Lip}\left(\mathbb{B}^{n+1}, \mathbb{H}^{n}\right)$ when $n \leq p<n+1$. To the contrary suppose that there is a sequence $g_{k} \in \operatorname{Lip}\left(\mathbb{B}^{n+1}, \mathbb{H}^{n}\right)$ such that

$$
g_{k} \rightarrow f \quad \text { in } W^{1, p}\left(\mathbb{B}^{n+1}, \mathbb{H}^{n}\right) .
$$

Clearly $f \in W^{1, p}\left(\mathbb{B}^{n+1}, \mathbb{R}^{2 n+1}\right)$ and $g_{k} \in \operatorname{Lip}\left(\mathbb{B}^{n+1}, \mathbb{R}^{2 n+1}\right)$. However, we cannot use Corollary 1.8, because we cannot assume that the mappings $g_{k}$ are uniformly bounded.

By Theorem 2.5. $\mathcal{H}_{c c}^{n+1}\left(g_{k}\left(\mathbb{B}^{n+1}\right)\right)=0$ for all $k$. Consequently, $\mathcal{H}^{n+1}\left(g_{k}\left(\mathbb{B}^{n+1}\right)\right)=$ 0 for all $k$ as well, since the identity map from $\mathbb{H}^{n}$ to $\mathbb{R}^{2 n+1}$ is locally Lipschitz. (Recall that $\mathcal{H}^{k}$ denotes the $k$-dimensional Euclidean Hausdorff measure.) 
Consequently, in order to arrive at a contradiction, it suffices to show that

$$
\mathcal{H}^{n+1}\left(g_{k}\left(\mathbb{B}^{n+1}\right)\right)>0 \quad \text { for sufficiently large } k \text {. }
$$

Let $\omega$ be the pullback by $\psi^{-1}$ of the volume form from $\partial \mathbb{B}^{n+1}=\mathbb{S}^{n}$ to $\psi\left(\mathbb{S}^{n}\right)$. We can assume that $\omega$ is a smooth compactly supported $n$-form on $\mathbb{R}^{2 n+1}$. We used a similar construction in the proof of Proposition 4.7.

By $\mathbb{S}^{n}(r)$ and $\mathbb{B}^{n+1}(r)$ we will denote the sphere and the ball of radius $r$ centered at the origin. Observe that since $\left.f\right|_{\mathbb{S}^{n}(r)}$ is just a rescaling of the map $\psi$, then

$$
\int_{\mathbb{S}^{n}(r)} f^{*} \omega=\mathcal{H}^{n}\left(\mathbb{S}^{n}\right)>0
$$

Let $K=\operatorname{supp} \omega$, let $S_{k}=\left\{x \in \mathbb{B}^{n+1}: g_{k}(x)-f(x) \in Z\right\}$ and let $E_{k}=S_{k} \cup g_{k}^{-1}(K)$. We claim that

$$
\left(\nabla g_{k}\right) \chi_{E_{k}} \rightarrow \nabla f \quad \text { in } L^{p}\left(\mathbb{B}^{n+1}\right) .
$$

Indeed, according to Lemma 6.5 $\nabla g_{k}=\nabla f$ a.e. in $S_{k}$, hence $\int_{S_{k}}\left|\nabla f-\nabla g_{k}\right|^{p}=0$.

Since the mappings $f$ and $\left.g_{k}\right|_{g_{k}^{-1}(K)}, k=1,2, \ldots$ are uniformly bounded Theorem 1.7 yields

$$
\int_{\mathbb{B}^{n+1} \backslash S_{k}}|\nabla f|^{p}+\left|\nabla g_{k}\right|^{p} \chi_{E_{k}} \leq C \int_{\mathbb{B}^{n+1} \backslash S_{k}}|\nabla f|_{\mathbb{H}}^{p}+\left|\nabla g_{k}\right|_{\mathbb{H}}^{p} \rightarrow 0 .
$$

Thus

$\int_{\mathbb{B}^{n+1}}\left|\nabla f-\left(\nabla g_{k}\right) \chi_{E_{k}}\right|^{p} \leq C\left(\int_{S_{k}}\left|\nabla f-\nabla g_{k}\right|^{p}+\int_{\mathbb{B}^{n+1} \backslash S_{k}}|\nabla f|^{p}+\left|\nabla g_{k}\right|^{p} \chi_{E_{k}}\right) \rightarrow 0$.

It follows from the Fubini theorem (again, see [27, pp. 189-190]) that we may select a subsequence of $\left(g_{k}\right)$, still denoted $\left(g_{k}\right)$, such that

$$
\left.\left.\left(\nabla g_{k}\right) \chi_{E_{k}}\right|_{\mathbb{S}^{n}(r)} \rightarrow \nabla f\right|_{\mathbb{S}^{n}(r)} \quad \text { in } L^{p}\left(\mathbb{S}^{n}(r)\right) \text { for a.e. } 0<r<1 .
$$

Fix such $r$. Since $K=\operatorname{supp} \omega, g_{k}^{*} \omega=0$ in $\mathbb{B}^{n+1} \backslash g_{k}^{-1}(K)$ and hence $g_{k}^{*} \omega=0$ in $\mathbb{B}^{n+1} \backslash E_{k}$. It follows from Lemma 4.9 that

$$
\int_{\mathbb{B}^{n+1}(r)} g_{k}^{*}(d \omega)=\int_{\mathbb{S}^{n}(r)} g_{k}^{*} \omega=\int_{\mathbb{S}^{n}(r) \cap E_{k}} g_{k}^{*} \omega
$$

and an application of Hölder's inequality (note that $g_{k}^{*} \omega, f^{*} \omega \in L^{p / n}\left(\mathbb{S}^{n}(r)\right)$ and $\frac{p}{n} \geq 1$ by assumption) yields

$$
\int_{\mathbb{S}^{n}(r) \cap E_{k}} g_{k}^{*} \omega \rightarrow \int_{\mathbb{S}^{n}(r)} f^{*} \omega=\mathcal{H}^{n}\left(\mathbb{S}^{n}\right)>0 .
$$

Hence

$$
\int_{\mathbb{B}^{n+1}(r)} g_{k}^{*}(d \omega)>0
$$

for sufficiently large $k$. Using the same argument involving the area formula as in the proof of Proposition 4.7 we conclude that

$$
\mathcal{H}^{n+1}\left(g_{k}\left(\mathbb{B}^{n+1}\right)\right) \geq \mathcal{H}^{n+1}\left(g_{k}\left(\mathbb{B}^{n+1}(r)\right)\right)>0,
$$

which proves (7.1). This completes the proof of Proposition 1.3 , 
Proof of Theorem 1.2(a). Suppose that $\operatorname{dim} M \geq n+1$. We need to prove that Lipschitz mappings Lip $\left(M, \mathbb{H}^{n}\right)$ are not dense in $W^{1, p}\left(M, \mathbb{H}^{n}\right)$ when $n \leq p<n+1$. This result will follow from Proposition 1.3 in a standard way; the same technique has already been used in the case of maps into manifolds in [8] and [27]. For the sake of completeness we provide the details.

It is easy to construct a smooth mapping $f: \mathbb{B}^{n+1} \rightarrow \mathbb{S}^{n}$ with two singular points such that $f$ restricted to small spheres centered at the singularities has degree +1 and -1 respectively and $f$ maps a neighborhood of the boundary of the ball $\mathbb{B}^{n+1}$ into a point. We can model the singularities on the radial projection mapping as in Proposition 1.3 so the mapping $f$ belongs to $W^{1, p}$. Now, let $g$ : $\mathbb{B}^{n+1} \times \mathbb{S}^{\operatorname{dim} M-n-1} \rightarrow \mathbb{S}^{n}$ be defined by $g(b, s)=f(b)$. We can embed the torus $\mathbb{B}^{n+1} \times \mathbb{S}^{\operatorname{dim} M-n-1}$ into the manifold $M$ and extend the mapping on the completion of this torus as a mapping into a point. Clearly, $g \in W^{1, p}\left(M, \mathbb{S}^{n}\right)$.

Now let $\psi: \mathbb{S}^{n} \rightarrow \mathbb{H}^{n}$ be a smooth and horizontal (hence bi-Lipschitz) embedding. We will prove that the mapping $\psi \circ g \in W^{1, p}\left(M, \mathbb{H}^{n}\right)$ cannot be approximated by Lipschitz mappings from $\operatorname{Lip}\left(M, \mathbb{H}^{n}\right)$. By way of contradiction suppose that $u_{k} \in \operatorname{Lip}\left(M, \mathbb{H}^{n}\right)$ converges to $\psi \circ f$ in the norm of $W^{1, p}$. In particular, $u_{k} \rightarrow \psi \circ g$ in $W^{1, p}\left(\mathbb{B}^{n+1} \times \mathbb{S}^{\operatorname{dim} M-n-1}, \mathbb{H}^{n}\right)$. It follows from Fubini's theorem that there is a subsequence of $\left(u_{k}\right)$, still denoted $\left(u_{k}\right)$, such that for a.e. $s \in \mathbb{S}^{\operatorname{dim} M-n-1}, u_{k}$ restricted to the slice $\mathbb{B}^{n+1} \times\{s\}$ converges to the corresponding restriction of $\psi \circ g$ in the Sobolev norm. Take such a slice and denote it simply by $\mathbb{B}^{n+1}$. Hence $u_{k}$ restricted to a ball (of dimension $n+1$ ) centered at the singularity of degree +1 converges to $\psi \circ g$ restricted to the same ball. This is, however, impossible as was demonstrated in Proposition 1.3 .

Proof of Theorem 1.2(b). Let $M$ be a compact Riemannian manifold of dimension at most $n$ and let $f \in W^{1, p}\left(M, \mathbb{H}^{n}\right)$. Then $\bar{f}=\kappa \circ f \in W^{1, p}\left(M, \ell^{\infty}\right)$. According to Lemma 5.6. $\|\bar{f}(x)-\bar{f}(y)\| \leq C d(x, y)(\mathcal{M}|\nabla \bar{f}|(x)+\mathcal{M}|\nabla \bar{f}|(y))$ for a.e. $x, y$. Let $E_{t}=\{x \in M: \mathcal{M}|\nabla \bar{f}|(x) \leq t\}$. The map $\left.\bar{f}\right|_{E_{t}}: E_{t} \rightarrow \ell^{\infty}$ is $C t$-Lipschitz, so $\left.f\right|_{E_{t}}: E_{t} \rightarrow \mathbb{H}^{n}$ is also. According to Theorem [2.6 there is a $C^{\prime} t$-Lipschitz map $f_{t}: M \rightarrow \mathbb{H}^{n}$ which coincides with $f$ on $E_{t}$. By the same argument as in the proof of Proposition [5.4, $f_{t} \rightarrow f$ in $W^{1, p}\left(M, \mathbb{H}^{n}\right)$.

\section{Sobolev maps into Grushin Planes}

The Grushin plane $G_{n}$ is the sub-Riemannian manifold whose underlying space is $M=\mathbb{R}^{2}$, with horizontal distribution $H M$ defined by the vector fields

$$
\xi_{1}=\frac{\partial}{\partial x}, \quad \xi_{2}=x^{n} \frac{\partial}{\partial y} .
$$

We note that this distribution does not have constant rank, however, it is bracket generating: after $n$ brackets we obtain $\left[\xi_{1},\left[\xi_{1},\left[\ldots, \xi_{2}\right] \cdots\right]=n ! \frac{\partial}{\partial y}\right.$. We define a sub-Riemannian (Carnot-Carathéodory) metric $d_{c c}$ on $M$ by declaring $\xi_{1}$ and $\xi_{2}$ to be an orthonormal basis at each point $(x, y) \in M$ with $x \neq 0$, and declaring $\xi_{1}$ to be normal at each point $(0, y) \in M$. We obtain a sub-Riemannian manifold $G_{n}$ of step $n+1$.

In this section we prove the following theorem.

Theorem 8.1. Equip $G_{n}$ with the Carnot-Carathéodory metric $d_{c c}$. Then Lipschitz mappings from $\mathbb{B}^{2}$ to $G_{n}$ are not dense in $W^{1, p}\left(\mathbb{B}^{2}, G_{n}\right)$ provided $1 \leq p<2$. 
For simplicity we consider only the case $G=G_{1}$. For $y_{1}>0, G$ contains infinitely many geodesics (local length minimizers) joining $(0,0)$ and $\left(0, y_{1}\right)$. See, e.g., 9, p. 275]. They are given by

$$
x_{m}(s)= \pm \sqrt{\frac{2 y_{1}}{m \pi}} \sin (m \pi s), \quad y_{m}(s)=y_{1}\left(s-\frac{\sin (2 m \pi s)}{2 m \pi}\right)
$$

for $m \in \mathbb{Z}^{+}$. When $m=1$ we obtain a single arc rising to $\left(0, y_{1}\right)$ in the first quadrant and another in the second quadrant.

Concatenating these two geodesics for $y_{1}=1$ yields a set which is bi-Lipschitz parameterized by $\mathbb{S}^{1}$. Let $\phi: \mathbb{S}^{1} \rightarrow G$ be a bi-Lipschitz mapping whose image is this union of geodesics. Let $u_{0}: \mathbb{B}^{2} \rightarrow \mathbb{S}^{1}$ be the cavitation map as in (1.1). Since $u_{0} \in W^{1, p}\left(\mathbb{B}^{2}, \mathbb{S}^{1}\right)$ for $p \in[1,2)$, the map

$$
f=\phi \circ u_{0}
$$

is in $W^{1, p}\left(\mathbb{B}^{2}, G\right)$ for the same range of $p$. We will show that $f$ cannot be approximated by Lipschitz functions from $\mathbb{B}^{2}$ to $G$.

The analog of the center $Z$ of the Heisenberg group is the vertical line $Y:=$ $\{(x, y) \in G: x=0\}$. The key technical result in the proof of Theorem 8.1 is the following lemma. Note that the restriction of the Hausdorff 2-measure in the metric $d_{c c}$ to the set $Y$ coincides (up to a constant) with the Euclidean length measure on $Y$.

Lemma 8.2. Let $f: \mathbb{B}^{2} \rightarrow G$ be a Lipschitz map. Then $\mathcal{H}_{c c}^{2}\left(f\left(\mathbb{B}^{2}\right) \cap Y\right)=0$.

Proof. Let $X=f^{-1}(Y) \subset \mathbb{B}^{2}$. As a substitute for the horizontal (Pansu) differential, we will use Kirchheim's metric differential [35]. It follows from Kirchheim's metric differentiability theorem [35. Theorem 2] that $f$ is almost everywhere metrically differentiable. That is, at a.e. point in $\mathbb{B}^{2}$ there exists a seminorm $m d f_{x}:=\|\cdot\|_{x}$ such that $d(f(x), f(y))-\|y-x\|_{x}=o(|y-x|$ as $y \rightarrow x$.

For a seminorm $s$ on $\mathbb{R}^{2}$, the Jacobian is defined as

$$
\mathbf{J}_{2}(s)=\frac{\pi}{\mathcal{H}^{2}(\{x: s(x) \leq 1\})} .
$$

By the area formula [3, Theorem 5.1],

$$
\int_{X} \mathbf{J}_{2}\left(m d f_{x}\right) d x=\int_{Y} \mathcal{H}^{0}\left(f^{-1}(y)\right) d \mathcal{H}_{c c}^{2}(y) .
$$

Hence $\mathcal{H}_{c c}^{2}\left(f\left(\mathbb{B}^{2}\right) \cap Y\right) \leq \int_{X} \mathbf{J}_{2}\left(m d f_{x}\right)$ and we claim that

$$
\mathbf{J}_{2}\left(m d f_{x}\right)=0 \quad \text { for a.e. } x \in X \text {. }
$$

To see why this claim holds, note that the identity map from $G$ to $\mathbb{R}^{2}$ is locally Lipschitz, so $f$ is still Lipschitz when considered as a map into $\mathbb{R}^{2}$. By Rademacher's theorem, the differential $d f$ exists a.e. At points where $d f$ exists, it is clear that $\mathbf{J}_{2}\left(m d f_{x}\right)=\operatorname{det}\left(d f_{x}\right)$. It remains to show that $d f$ is singular for a.e. $x \in X$.

Suppose $d f_{x}$ is nonsingular at $x \in X$ and let $v:=\left(d f_{x}\right)^{-1}\left(\frac{\partial}{\partial y}\right)$. Then $\mid f(x+t v)-$ $f(x)-\left.(0, t)\right|_{E}$ is $O\left(t^{2}\right)$, whence

$$
d_{c c}(f(x+t v), f(x)+(0, t))=O(t)
$$

as $t \rightarrow 0$, by (2.8). On the other hand, there exists a constant $c>0$ so that

$$
d_{c c}(f(x), f(x)+(0, t))=c \sqrt{t}
$$


for all $t>0$, since $x \in X$ and $\left.d_{c c}\right|_{Y}$ is a multiple of $\sqrt{\left.d_{E}\right|_{Y}}$. We conclude that

$$
d_{c c}(f(x+t v), f(x)) \geq c \sqrt{t}-O(t)
$$

as $t \rightarrow 0$, which contradicts the assumption that $f$ is Lipschitz.

In conclusion, $d f_{x}$ is defined a.e. and is singular where defined. Using the area formula, the proof is complete.

We next reduce the question of Sobolev convergence to one about Euclidean targets.

Lemma 8.3. If $f_{k} \rightarrow f$ in $W^{1, p}\left(\mathbb{B}^{2}, G\right)$, then $f_{k} \rightarrow f$ in $W^{1, p}\left(\mathbb{B}^{2}, \mathbb{R}^{2}\right)$.

Proof. The same method applies here as in the case of Heisenberg targets. The only difference is that for $p, q \in G$, the phrase " $p$ and $q$ are vertically separated" must be interpreted " $p, q \in Y$ ". Since the metric is Riemannian outside of $Y$, it is $C^{\infty}$ on $G \backslash Y$.

Proof of Theorem 8.1. Let $f$ be the map in (8.2). Suppose there exists a sequence of Lipschitz maps $f_{k}: \mathbb{B}^{2} \rightarrow G$ approximating $f$ in $W^{1, p}\left(\mathbb{B}^{2}, G\right)$. By Lemma 8.3. $f_{k}$ converges to $f$ in $W^{1, p}\left(\mathbb{B}^{2}, \mathbb{R}^{2}\right)$. Fix $\epsilon>0$ sufficiently small (to be determined later) and fix $k$ sufficiently large so that

$$
\left\|f-f_{k}\right\|_{W^{1, p}\left(\mathbb{B}^{2}, \mathbb{R}^{2}\right)}<\epsilon .
$$

Let $g=f-f_{k}$. By Fubini's theorem, $\left.g\right|_{S_{r}} \in W^{1, p}\left(S_{r}, \mathbb{R}^{2}\right)$ for almost every $r \in$ $(0,1]$, where $S_{r}$ denotes the circle of radius $r$ centered at the origin. Furthermore, there exists $r \in\left(1-2^{-p}, 1\right)$ so that the Sobolev norm of $\left.g\right|_{S_{r}}$ is less than $2 \epsilon$. We claim that $f_{k}\left(B_{r}\right)$ intersects $Y$ in a set of positive length (and hence positive $\mathcal{H}_{c c}^{2}$ measure). Here $B_{r}$ denotes the disc of radius $r$ centered at the origin.

Since $\|g\|_{L^{p}\left(S_{r}\right)}<2 \epsilon$, there exists $x_{0} \in S_{r}$ with $\left|g\left(x_{0}\right)\right|<2 \epsilon(2 \pi r)^{-1 / p}$. Furthermore, $\|d g\|_{L^{p}\left(S_{r}\right)}<2 \epsilon$. Applying the Fundamental Theorem of Calculus along $S_{r}$ gives

$$
\|g\|_{L^{\infty}\left(S_{r}\right)} \leq\left|g\left(x_{0}\right)\right|+\|d g\|_{L^{1}\left(S_{r}\right)} \leq 2 \epsilon\left((2 \pi r)^{-1 / p}+(2 \pi r)^{1-1 / p}\right) .
$$

Since $r$ is bounded away from zero, it is clear that by choosing $\epsilon$ sufficiently small (depending only on $p$ ) we may ensure that $\left\|f_{k}-f\right\|_{L^{\infty}\left(S_{r}\right)}=\|g\|_{L^{\infty}\left(S_{r}\right)}<\frac{1}{2}$. We conclude that there exists a nondegenerate interval $J$ contained in $Y$ so that the winding number of $f_{k}\left(S_{r}\right)$ around each point of $J$ is nonzero. By a degree theory argument similar to those used previously, we conclude that $(0, y) \in f_{k}\left(B_{r}\right)$ for all $(0, y) \in J$. This completes the proof of the claim. Since $f_{k}$ is Lipschitz, the validity of this claim violates Lemma 8.2. Hence the approximating sequence $f_{k}$ cannot exist and the proof of Theorem 8.1 is complete.

Remark 8.4. The proof of Theorem 8.1 relied on the construction of a bi-Lipschitz embedding of $\mathbb{S}^{1}$ into $G$ whose image enclosed a nontrivial segment on the $y$-axis $Y$. Such a bi-Lipschitz embedding admits no Lipschitz extension to $\mathbb{B}^{2}$. By way of contrast, we have the following

Theorem 8.5. Let $\varphi: \mathbb{S}^{1} \rightarrow G$ be an L-Lipschitz map with $\varphi\left(\mathbb{S}^{1}\right) \cap Y=\emptyset$. Then $\varphi$ admits an L-Lipschitz extension $\tilde{\varphi}: \mathbb{B}^{2} \rightarrow G$.

Theorem 8.5 follows from a generalized form of Kirszbraun's theorem proved by Lang and Schroeder [37, Theorem A]. For simplicity we only state a special case of the Lang-Schroeder theorem sufficient for our purposes. See also 36. Theorem 
1.3] for a related result (which implies a weaker form of Theorem 8.5 where the Lipschitz constant of the extension may be larger than $L$ ).

Theorem 8.6 (Lang-Schroeder). Let $M$ and $N$ be Riemannian manifolds (possibly with boundary). Assume that $M$ has all sectional curvatures bounded below by some $k \in \mathbb{R}$, while $N$ is complete and has all sectional curvatures bounded above by the same $k$. Then every 1-Lipschitz map $f: A \rightarrow N, A \subset M$, admits a 1-Lipschitz extension $\tilde{f}: M \rightarrow N$.

Proof of Theorem 8.5. Let $\varphi: \mathbb{S}^{1} \rightarrow G$ be $L$-Lipschitz with $\varphi\left(\mathbb{S}^{1}\right) \cap Y=\emptyset$. By rescaling the metric in $\mathbb{R}^{2}$ if necessary, we may assume without loss of generality that $L=1$.

Observe that $\varphi\left(\mathbb{S}^{1}\right)$ is contained in $\{(x, y) \in G:|x| \geq \epsilon\}$ for some $\epsilon>0$. In particular, $\varphi(\mathbb{S})$ lies completely within a Riemannian component of $G$. We next compute the curvature of such a component. The Riemannian metric in $\{(x, y)$ : $x>0\}$ is given by the length element $d s^{2}=d x^{2}+x^{-2} d y^{2}$. Using Brioschi's formula

$$
K=\frac{1}{2 \sqrt{E G}}\left[\left(\frac{G_{x}}{\sqrt{E G}}\right)_{x}+\left(\frac{E_{y}}{\sqrt{E G}}\right)_{y}\right]
$$

for the (Gauss) curvature of a Riemannian metric $d s^{2}=E d x^{2}+G d y^{2}$ with $E=1$ and $G=x^{-2}$, we obtain $K=-2 x^{-2}$. Thus $K \leq-2 \epsilon^{-2}<0$ on $\{(x, y) \in G: x \geq \epsilon\}$. A similar computation applies in $\{(x, y) \in G: x \leq-\epsilon\}$.

It remains to show that the set $\{(x, y) \in G: x \geq \epsilon\}$, equipped with the aforementioned Riemannian metric, is complete and geodesic. The latter assertion follows from the explicit form (8.1) for the geodesics. Since $\{(x, y) \in G: x \geq \epsilon\}$ is locally compact, it is proper and hence complete. The proof of Theorem 8.5 is finished.

Question 8.7. Does every Lipschitz map $\varphi: \mathbb{S}^{1} \rightarrow G$ whose image encloses no segment on the $y$-axis extend to a Lipschitz map of $\mathbb{B}^{2}$ into $G$ ?

\section{ACKNOWLEDGEMENTS}

We thank the referee for a careful reading of the paper and for helpful remarks. In particular, we are grateful to the referee for insisting that we include more detail in the proof of Theorem 4.11, this request led us to articulate stronger conclusions regarding the structure of the Lipschitz homotopy group $\pi_{n}^{\text {Lip }}\left(\mathbb{H}^{n}\right)$ than we had originally deduced. See Remark 4.10 for details.

\section{REFERENCES}

[1] Emilio Acerbi and Nicola Fusco, An approximation lemma for $W^{1, p}$ functions, Material instabilities in continuum mechanics (Edinburgh, 1985-1986), Oxford Sci. Publ., Oxford Univ. Press, New York, 1988, pp. 1-5. MR970512 (89m:46060)

[2] D. Allcock, An isoperimetric inequality for the Heisenberg groups, Geom. Funct. Anal. 8 (1998), no. 2, 219-233, DOI 10.1007/s000390050053. MR1616147 (99g:53038)

[3] Luigi Ambrosio and Bernd Kirchheim, Rectifiable sets in metric and Banach spaces, Math. Ann. 318 (2000), no. 3, 527-555, DOI 10.1007/s002080000122. MR1800768 (2003a:28009)

[4] L. Ambrosio and S. Rigot, Optimal mass transportation in the Heisenberg group, J. Funct. Anal. 208 (2004), no. 2, 261-301, DOI 10.1016/S0022-1236(03)00019-3. MR2035027 (2004m:49107)

[5] Zoltán M. Balogh and Katrin S. Fässler, Rectifiability and Lipschitz extensions into the Heisenberg group, Math. Z. 263 (2009), no. 3, 673-683, DOI 10.1007/s00209-008-0437-z. MR2545863 (2010j:53049) 
[6] André Bellaïche, The tangent space in sub-Riemannian geometry, Sub-Riemannian geometry, Progr. Math., vol. 144, Birkhäuser, Basel, 1996, pp. 1-78, DOI 10.1007/978-3-0348-9210-0_1. MR.1421822 (98a:53108)

[7] Fabrice Bethuel, The approximation problem for Sobolev maps between two manifolds, Acta Math. 167 (1991), no. 3-4, 153-206, DOI 10.1007/BF02392449. MR1120602 (92f:58023)

[8] Fabrice Bethuel and Xiao Min Zheng, Density of smooth functions between two manifolds in Sobolev spaces, J. Funct. Anal. 80 (1988), no. 1, 60-75, DOI 10.1016/0022-1236(88)90065-1. MR.960223 (89i:58015)

[9] Ovidiu Calin and Der-Chen Chang, Sub-Riemannian Geometry, Encyclopedia of Mathematics and its Applications, vol. 126, Cambridge University Press, Cambridge, 2009. General theory and examples. MR2503811 (2010a:53042)

[10] J. W. Cannon, G. R. Conner, and Andreas Zastrow, One-dimensional sets and planar sets are aspherical, Topology Appl. 120 (2002), no. 1-2, 23-45, DOI 10.1016/S0166-8641(01)00005-0. In memory of T. Benny Rushing. MR 1895481 (2003g:57028)

[11] Luca Capogna and Fang-Hua Lin, Legendrian energy minimizers. I. Heisenberg group target, Calc. Var. Partial Differential Equations 12 (2001), no. 2, 145-171, DOI 10.1007/PL00009910. MR.1821235 (2002k:58032)

[12] Luca Capogna, Donatella Danielli, Scott D. Pauls, and Jeremy T. Tyson, An Introduction to the Heisenberg Group and the Sub-Riemannian Isoperimetric Problem, Progress in Mathematics, vol. 259, Birkhäuser Verlag, Basel, 2007. MR.2312336 (2009a:53053)

[13] Jeff Cheeger and Bruce Kleiner, Differentiability of Lipschitz maps from metric measure spaces to Banach spaces with the Radon-Nikodým property, Geom. Funct. Anal. 19 (2009), no. 4, 1017-1028, DOI 10.1007/s00039-009-0030-6. MR2570313 (2011c:30138)

[14] Jeff Cheeger and Bruce Kleiner, Generalized differential and bi-Lipschitz nonembedding in $L^{1}$ (English, with English and French summaries), C. R. Math. Acad. Sci. Paris 343 (2006), no. 5, 297-301, DOI 10.1016/j.crma.2006.07.001. MR.2253046 (2007k:58014)

[15] J. Diestel and J. J. Uhl Jr., Vector Measures, American Mathematical Society, Providence, R.I., 1977. With a foreword by B. J. Pettis; Mathematical Surveys, No. 15. MR0453964 (56 \#12216)

[16] J. Eells and L. Lemaire, A report on harmonic maps, Bull. London Math. Soc. 10 (1978), no. 1, 1-68, DOI 10.1112/blms/10.1.1. MR495450(82b:58033)

[17] Tobias Ekholm, John Etnyre, and Michael Sullivan, Non-isotopic Legendrian submanifolds in $\mathbb{R}^{2 n+1}$, J. Differential Geom. 71 (2005), no. 1, 85-128. MR2191769 (2006i:53119)

[18] Lawrence C. Evans and Ronald F. Gariepy, Measure Theory and Fine Properties of Functions, Studies in Advanced Mathematics, CRC Press, Boca Raton, FL, 1992. MR1158660 (93f:28001)

[19] G. B. Folland and Elias M. Stein, Hardy Spaces on Homogeneous Groups, Mathematical Notes, vol. 28, Princeton University Press, Princeton, N.J., University of Tokyo Press, Tokyo, 1982. MR657581 (84h:43027)

[20] Mikhael Gromov, Partial Differential Relations, Ergebnisse der Mathematik und ihrer Grenzgebiete (3) [Results in Mathematics and Related Areas (3)], vol. 9, Springer-Verlag, Berlin, 1986. MR864505 (90a:58201)

[21] Mikhael Gromov, Carnot-Carathéodory Spaces Seen from Within, Sub-Riemannian geometry, Progr. Math., vol. 144, Birkhäuser, Basel, 1996, pp. 79-323. MR1421823 (2000f:53034)

[22] Piotr Hajłasz, Approximation of Sobolev mappings, Nonlinear Anal. 22 (1994), no. 12, 15791591, DOI 10.1016/0362-546X(94)90190-2. MR1285094(96a:58030)

[23] Piotr Hajłasz, Sobolev spaces on an arbitrary metric space, Potential Anal. 5 (1996), no. 4, 403-415, DOI 10.1007/BF00275475. MR 1401074 (97f:46050)

[24] Piotr Hajłasz, Sobolev mappings: Lipschitz density is not a bi-Lipschitz invariant of the target, Geom. Funct. Anal. 17 (2007), no. 2, 435-467, DOI 10.1007/s00039-007-0594-y. MR2322491 (2008e:58012)

[25] Piotr Hajłasz, Density of Lipschitz mappings in the class of Sobolev mappings between metric spaces, Math. Ann. 343 (2009), no. 4, 801-823, DOI 10.1007/s00208-008-0291-7. MR2471602 (2009j:58014)

[26] Piotr Hajłasz, Sobolev mappings: Lipschitz density is not an isometric invariant of the target, Int. Math. Res. Not. IMRN 12 (2011), 2794-2809, DOI 10.1093/imrn/rnq183. MR2806594 (2012d:58011) 
[27] Piotr Hajłasz, Sobolev mappings between manifolds and metric spaces, Sobolev Spaces in Mathematics. I, Int. Math. Ser. (N. Y.), vol. 8, Springer, New York, 2009, pp. 185-222, DOI 10.1007/978-0-387-85648-3_7. MR2508843 (2010j:46069)

[28] Piotr Hajłasz and Pekka Koskela, Sobolev met Poincaré, Mem. Amer. Math. Soc. 145 (2000), no. 688, x+101, DOI 10.1090/memo/0688. MR.1683160 (2000j:46063)

[29] Piotr Hajłasz, Armin Schikorra, and Jeremy T. Tyson, Homotopy groups of spheres and Lipschitz homotopy groups of Heisenberg groups, Geom. Funct. Anal. 24 (2014), no. 1, 245268, DOI 10.1007/s00039-014-0261-z. MR.3177382

[30] Piotr Hajłasz and Jeremy T. Tyson, Sobolev Peano cubes, Michigan Math. J. 56 (2008), no. 3, 687-702, DOI 10.1307/mmj/1231770368. MR2490654 (2010c:46081)

[31] Fengbo Hang and Fanghua Lin, Topology of Sobolev mappings. II, Acta Math. 191 (2003), no. 1, 55-107, DOI 10.1007/BF02392696. MR2020419 (2005m:58023)

[32] Allen Hatcher, Algebraic Topology, Cambridge University Press, Cambridge, 2002. MR.1867354(2002k:55001)

[33] Juha Heinonen, Lectures on Lipschitz Analysis, Report. University of Jyväskylä Department of Mathematics and Statistics, vol. 100, University of Jyväskylä, Jyväskylä, 2005. MR2177410 (2006k:49111)

[34] Juha Heinonen, Pekka Koskela, Nageswari Shanmugalingam, and Jeremy T. Tyson, Sobolev classes of Banach space-valued functions and quasiconformal mappings, J. Anal. Math. 85 (2001), 87-139, DOI 10.1007/BF02788076. MR.1869604 (2002k:46090)

[35] Bernd Kirchheim, Rectifiable metric spaces: local structure and regularity of the Hausdorff measure, Proc. Amer. Math. Soc. 121 (1994), no. 1, 113-123, DOI 10.2307/2160371. MR.1189747 (94g:28013)

[36] U. Lang, B. Pavlović, and V. Schroeder, Extensions of Lipschitz maps into Hadamard spaces, Geom. Funct. Anal. 10 (2000), no. 6, 1527-1553, DOI 10.1007/PL00001660. MR1810752 (2002d:53054)

[37] U. Lang and V. Schroeder, Kirszbraun's theorem and metric spaces of bounded curvature, Geom. Funct. Anal. 7 (1997), no. 3, 535-560, DOI 10.1007/s000390050018. MR 1466337 (98d:53062)

[38] Valentino Magnani, Elements of Geometric Measure Theory on Sub-Riemannian Groups, Scuola Normale Superiore, Pisa, 2002. MR2115223 (2005m:28002)

[39] Valentino Magnani, Unrectifiability and rigidity in stratified groups, Arch. Math. (Basel) 83 (2004), no. 6, 568-576, DOI 10.1007/s00013-004-1057-4. MR2105335 (2005i:53033)

[40] Valery Marenich, Geodesics in Heisenberg groups, Geom. Dedicata 66 (1997), no. 2, 175-185, DOI 10.1023/A:1004916117293. MR1458790 (98h:53068)

[41] Pertti Mattila, Geometry of Sets and Measures in Euclidean Spaces, Cambridge Studies in Advanced Mathematics, vol. 44, Cambridge University Press, Cambridge, 1995. Fractals and rectifiability. MR $1333890(96 \mathrm{~h}: 28006)$

[42] Richard Montgomery, A Tour of Subriemannian Geometries, Their Geodesics and Applications, Mathematical Surveys and Monographs, vol. 91, American Mathematical Society, Providence, RI, 2002. MR.1867362 (2002m:53045)

[43] Roberto Monti, Some properties of Carnot-Carathéodory balls in the Heisenberg group (English, with English and Italian summaries), Atti Accad. Naz. Lincei Cl. Sci. Fis. Mat. Natur. Rend. Lincei (9) Mat. Appl. 11 (2000), no. 3, 155-167 (2001). MR.1841689 (2002c:53048)

[44] Alexander Nagel, Elias M. Stein, and Stephen Wainger, Balls and metrics defined by vector fields. I. Basic properties, Acta Math. 155 (1985), no. 1-2, 103-147, DOI 10.1007/BF02392539. MR793239 (86k:46049)

[45] Pierre Pansu, Métriques de Carnot-Carathéodory et quasiisométries des espaces symétriques de rang un (French, with English summary), Ann. of Math. (2) 129 (1989), no. 1, 1-60, DOI 10.2307/1971484. MR 979599 (90e:53058)

[46] Yu. G. Reshetnyak, Sobolev classes of functions with values in a metric space (Russian, with Russian summary), Sibirsk. Mat. Zh. 38 (1997), no. 3, 657-675, iii-iv, DOI 10.1007/BF02683844; English transl., Siberian Math. J. 38 (1997), no. 3, 567-583. MR 1457485 (98h:46031)

[47] Séverine Rigot and Stefan Wenger, Lipschitz non-extension theorems into jet space Carnot groups, Int. Math. Res. Not. IMRN 18 (2010), 3633-3648, DOI 10.1093/imrn/rnq023. MR2725507 (2011f:53056) 
[48] Richard Schoen and Karen Uhlenbeck, Boundary regularity and the Dirichlet problem for harmonic maps, J. Differential Geom. 18 (1983), no. 2, 253-268. MR710054 (85b:58037)

[49] Richard Schoen and Karen Uhlenbeck, Approximation theorems for Sobolev mappings, unpublished.

[50] Stefan Wenger and Robert Young, Lipschitz extensions into jet space Carnot groups, Math. Res. Lett. 17 (2010), no. 6, 1137-1149, DOI 10.4310/MRL.2010.v17.n6.a12. MR2729637 (2011j:53048)

[51] Stefan Wenger and Robert Young, Lipschitz homotopy groups of the Heisenberg groups, Geom. Funct. Anal. 24 (2014), no. 1, 387-402, DOI 10.1007/s00039-014-0264-9. MR3177387

[52] Kōsaku Yosida, Functional analysis, Classics in Mathematics, Springer-Verlag, Berlin, 1995. Reprint of the sixth (1980) edition. MR1336382 (96a:46001)

Department of Mathematics, University of Illinois at Urbana-Champaign, 1409 West Green St., Urbana, Illinois 61801

E-mail address: ndejarne@illinois.edu

Department of Mathematics, University of Pittsburgh, 301 Thackeray Hall, PittsBurgh, Pennsylvania 15260

E-mail address: hajlasz@pitt.edu

Department of Mathematics, University of Illinois at Urbana-Champaign, 1409 West Green St., Urbana, Illinois 61801

E-mail address: lukyane2@illinois.edu

Department of Mathematics, University of Illinois at Urbana-Champaign, 1409 West Green St., Urbana, Illinois 61801

E-mail address: tyson@math.uiuc.edu 